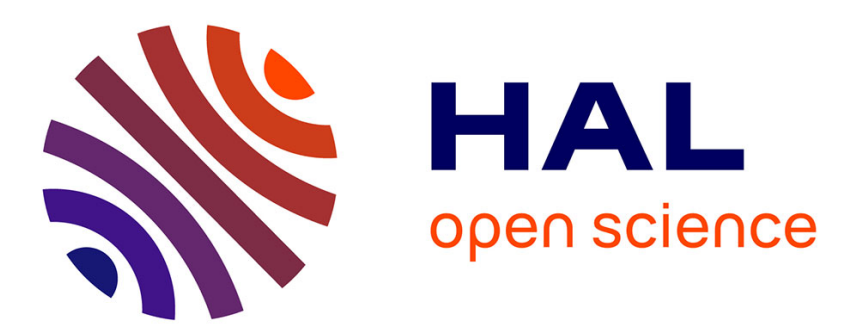

\title{
Le temps consacré aux enfants : les enseignantes et enseignants se distinguent-ils des autres diplômés de l'enseignement supérieur?
}

Muriel Letrait, Fanny Salane

\section{- To cite this version:}

Muriel Letrait, Fanny Salane. Le temps consacré aux enfants: les enseignantes et enseignants se distinguent-ils des autres diplômés de l'enseignement supérieur?. Economie et Statistique / Economics and Statistics, 2015, Dispositifs sociaux et fiscaux en faveur des familles - Lien entre diplôme et insertion professionnelle - Dossier : "Emploi du temps" 478 (1), pp.243-271. 10.3406/estat.2015.10564 . hal-01410620

\section{HAL Id: hal-01410620 \\ https://hal.parisnanterre.fr/hal-01410620}

Submitted on 30 Nov 2017

HAL is a multi-disciplinary open access archive for the deposit and dissemination of scientific research documents, whether they are published or not. The documents may come from teaching and research institutions in France or abroad, or from public or private research centers.
L'archive ouverte pluridisciplinaire HAL, est destinée au dépôt et à la diffusion de documents scientifiques de niveau recherche, publiés ou non, émanant des établissements d'enseignement et de recherche français ou étrangers, des laboratoires publics ou privés. 


\title{
Le temps consacré aux enfants : les enseignantes et enseignants se distinguent-ils des autres diplômés de l'enseignement supérieur?
}

\author{
Muriel Letrait * et Fanny Salane **
}

Les enfants d'enseignants sont généralement les mieux situés dans la course scolaire, y compris lorsqu'ils sont comparés aux enfants de cadres. Une des explications à cet effet « parent enseignant » est l'organisation temporelle spécifique de cette profession, et la synchronisation des temps familiaux qu'elle permet.

Cet article, à partir de l'exploitation de l'enquête Emploi du Temps 2010 et du module spécifique « Enseignants », analyse le temps parental et sa répartition sexuée chez les enseignants et les diplômés non enseignants. Trois temps ont été étudiés : le temps total consacré aux enfants, le temps consacré uniquement aux jeux et à l'instruction, et le temps consacré uniquement aux activités de soins et d'accompagnement. En comparant ces types de temps, la recherche met en évidence que les logiques de genre, de compétence et de disponibilité se combinent.

En effet, les enseignants sont plus souvent et plus longtemps présents à leur domicile que les autres diplômés du supérieur, et consacrent globalement plus de temps à leurs enfants que ces derniers. Si on compare les hommes entre eux, et les femmes entre elles, le fait d'être enseignant entraîne une augmentation du temps consacré aux enfants les jours travaillés. Pour les femmes, ceci est vrai quel que soit le type de temps étudié. Pour les hommes, ceci est constaté uniquement pour le temps voué aux jeux et à l'instruction. Ces différences entre enseignants et autres diplômés du supérieur ne sont pas compensées les jours de repos.

La profession d'enseignant n'apparaît cependant pas remettre en cause l'inégale répartition sexuée des tâches liées aux enfants. Les hommes enseignants sont certes plus disponibles et participent plus aux activités de jeux et d'instruction les jours de travail que les femmes diplômés non-enseignantes, mais les femmes, enseignantes ou non, sont toujours celles qui consacrent le plus de temps aux activités de soins et d'accompagnement, quel que soit le type de jour étudié.

Codes JEL : J13, J16, J22.

Mots clés : parents enseignants, parents diplômés du supérieur, temps parental, effet « parent enseignant ».

\footnotetext{
* Muriel Letrait est ingénieure d'études statisticienne (Cerlis, Université Paris Descartes, Sorbonne Paris Cité).

** Fanny Salane est maître de conférences en sciences de l'éducation (CREF, Université Paris Ouest Nanterre - La Défense).
}

Remerciements : les auteures remercient les relecteurs/relectrices pour leurs conseils précieux et leurs remarques constructives sur la première version de l'article. Elles sont également reconnaissantes au groupe de travail " Emploi du temps » de l'Insee qui a permis, par des discussions et des échanges lors de la présentation des premiers résultats en mars 2013 notamment, de faire évoluer la recherche et de l'améliorer. 


\section{Les enfants d'enseignants, ces « chouchous » de l'école ${ }^{1}$..}

L'école et la construction d'une trajectoire de réussite sont aujourd'hui au centre des préoccupations parentales, et plus globalement, sociétales. Dans cette course scolaire, la position des enfants d'enseignants est singulière. En ce qui concerne les niveaux primaire et secondaire, ce sont en effet ceux qui réussissent le mieux et qui ont le plus de chances de faire un parcours scolaire « sans faute ». Ils redoublent et décrochent moins souvent que les enfants d'autres catégories sociales, s'orientent plus souvent dans des filières générales, vont plus loin et obtiennent des diplômes plus élevés et valorisés. Ils sont plus nombreux à poursuivre leurs études dans le supérieur et ont plus de chances d'accéder à des filières prestigieuses et reconnues.

Ainsi, le Repères et références statistiques sur les enseignements, la formation et la recherche (RERS) de 2012 montre que 15,2\% des filles d'ouvriers entrent en sixième avec du retard contre seulement $3 \%$ de filles de cadres et 2,5\% de filles d'enseignants. En ce qui concerne les garçons, on obtient respectivement 18,5\%, $4,3 \%$ et $3,5 \%$. L'étude des lauréats au diplôme du brevet des collèges en 2011 va dans le même sens : 77,7 \% des enfants d'ouvriers obtiennent ce diplôme, contre 89,5 \% des enfants de professions intermédiaires (et plus précisément 95,5\% des enfants d'instituteurs et assimilés), et $95,4 \%$ des enfants de professions intellectuelles supérieures (et parmi celles-ci, 96,9\% des enfants de professeurs et assimilés).

Le constat d'écarts de performances scolaires ne s'établit donc pas seulement entre milieux sociaux contrastés : si on les compare à des catégories socioprofessionnelles fortement dotées (cadres et professions intellectuelles supérieures), on remarque que les enfants d'enseignants conservent certains avantages, même si les différences paraissent ténues.

Parmi les élèves entrés en $6^{\mathrm{e}}$ en $1995,90,6 \%$ des enfants d'enseignants (professeurs et instituteurs) ont obtenu le baccalauréat, contre $87,6 \%$ des enfants de cadres. Plus précisément, en ce qui concerne le baccalauréat général et technologique, c'est le cas de $86,6 \%$ des enfants d'enseignants (entrés en $6^{\mathrm{e}}$ en 1995) et de $84,2 \%$ des enfants de cadres (Vanhoeffelen, 2010). Seuls 52,9\% d'enfants d'ouvriers qualifiés et $40,7 \%$ d'enfants d'ouvriers non qualifiés sont concernés.
De même, les enfants d'enseignants sont surreprésentés en filière $\mathrm{S}$. Or la série du baccalauréat joue un rôle capital dans l'orientation des bacheliers, contribuant au renforcement du marquage social des publics de chaque discipline du supérieur (Sautory, 2007). Pour l'enseignement supérieur, la surreprésentation des enfants d'enseignants persiste (DEP, 2003) mais est moins prégnante, ces derniers gardant toutefois un très léger avantage à l'université et dans les classes préparatoires aux grandes écoles littéraires (Sautory, 2007 ; Bouhia, 2005). Les enfants de cadres sont eux surreprésentés dans les classes préparatoires économiques et sociales (Sautory, 2007)2.

Nous pourrions multiplier les constats ; les données disponibles mettent en évidence que les enfants d'enseignants semblent toujours mieux positionnés dans l'espace scolaire que les enfants de cadres et professions intellectuelles supérieures, aux caractéristiques sociales pourtant proches, que ce soit au niveau primaire ou secondaire ${ }^{3}$.

\section{... « chouchoutés » par leurs parents ?}

Quelles sont les différentes explications que les chercheurs avancent à cet effet « parent enseignant », pour reprendre les termes de Da-Costa Lasne (2012)?

Si peu de chercheurs se sont finalement penchés sur les spécificités des enseignants en tant que parents, beaucoup de sociologues de l'éducation ont travaillé sur les explications de la meilleure réussite scolaire des enfants issus des classes supérieures.

Tout d'abord, c'est la possession de capitaux scolaire, social, mais surtout culturel et économique, qui apparaît comme un facteur

1. Cette expression fait référence au titre d'un article du journal Le Monde daté du 8 mai 2013, de Mattea Battaglia : "Enfants d'enseignants, ces "chouchous" de l'école".

2. Il est alors possible que l'insertion professionnelle des enfants d'enseignants et des enfants de cadres, et donc le rendement de leurs diplômes, soient différents.

3. Les enfants d'enseignants, dans ces études, sont en fait majoritairement des enfants dont le père est enseignant puisque, généralement, seule la profession du père est prise en compte. Toutefois, les hommes enseignants étant en forte proportion en couple avec des enseignantes - c'est le cas de plus de la moitié des enseignants du primaire, et d'un peu plus du tiers des enseignants du secondaire (Farges, 2011) - ces enfants sont souvent également des enfants d'enseignantes. Par ailleurs, il est vraisemblable qu'un certain nombre d'enfants considérés comme enfants de cadres dans ces études (car leur père est cadre) soient enfants de mère enseignante. Dans le présent article, le terme "enseignants ", utilisé dans le but d'alléger le texte, recouvrira les enseignantes et les enseignants. 
discriminant dans la construction d'une trajectoire scolaire de réussite (Bourdieu et Passeron, 1964). Ainsi la possession d'un capital culturel, notamment à «l'état incorporé, c'est-à-dire sous la forme de dispositions durables de l'organisme " (Bourdieu, 1979, p. 3) - qui se traduirait par une plus grande " docilité » scolaire par exemple - expliquerait les écarts de réussite scolaire.

Mais ceci ne permet pas vraiment de comprendre en quoi les enseignants sont spécifiques par rapport à d'autres parents, également fortement dotés en capitaux. Ce serait plus précisément la connivence culturelle et la familiarité des enseignants avec le monde scolaire, qui leur permettraient de répondre aux attentes implicites de l'institution et de mettre en place des «stratégies » avisées (Baudelot et Establet, 2009 ; Thin, 1998 ; Héran, 1996). Par exemple, les enseignants sont ceux qui bénéficient le plus des possibilités offertes par la désectorisation, parce qu'ils sont les plus à même "d'avoir les informations pertinentes et les stratégies adéquates pour en faire un instrument important de gestion de la carrière scolaire de leurs enfants » (Henriot-Van Zanten, 1996, p. 129). Par ailleurs, les styles éducatifs transmis et valorisés dans le monde enseignant, et dans le monde des professions moyennes et supérieures intellectuelles en général, seraient plus en adéquation avec les attentes et les normes du monde scolaire : argumentation, dialogue, négociation plutôt qu'imposition, autocontrainte plutôt que contrainte (Van Zanten, 2009).

Plus globalement, ce serait les pratiques parentales des enseignants, qui incluent donc les stratégies scolaires, mais également les types d'activités extrascolaires (partagées ou non avec son enfant), de loisirs, de lectures, de discussions, qui constitueraient un terrain plus favorable à la réussite scolaire des enfants d'enseignants (Da-Costa Lasne, 2012).

Une dernière caractéristique de ce groupe professionnel, qui les distingue des autres diplômés du supérieur en activité, concerne les contraintes temporelles : les emplois du temps des parents enseignants et de leurs enfants sont tous les deux structurés autour du temps scolaire, ce qui permet une co-présence importante au domicile et, par conséquent, une régulation des activités enfantines (Henri-Panabière, 2010 ; Millet et Thin, 2005 ; Chenu, 2002), ainsi qu'un partage d'activités communes plus souvent que chez d'autres catégories sociales.
L'enquête Emploi du temps de 2010, qui a la spécificité de comporter un module « Enseignants $»^{4}$ à la demande de la DEPP (Ministère de l'éducation nationale), donne la possibilité d'explorer ce dernier point, en examinant l'usage du temps par les parents enseignants, comparé à celui d'autres parents également diplômés. L'enquête ne permet pas d'évaluer à quel point la plus forte disponibilité parentale des enseignants contribue à la réussite scolaire des enfants, mais elle permet de chiffrer cette plus grande disponibilité et aussi d'étudier comment elle se différencie entre femmes et hommes enseignants. Il est en effet largement démontré que les femmes consacrent beaucoup plus de temps aux tâches parentales (Ponthieux et Schreiber, 2006 ; Anxo, Flood et Kocoglu, 2002 ; Algava, 2002). Qu'en est-il lorsque l'un et/ou l'autre des deux conjoints sont enseignants?

Dans cet article, nous nous penchons donc plus précisément sur l'usage du temps parental par les enseignants. Consacrent-ils plus de temps à leurs enfants, et pour quel type d'activités ? L'intérêt est de les comparer à des parents fortement diplômés, afin d'évaluer si leurs usages du temps parental sont différents, et en quoi. Par ailleurs, le temps consacré est-il différent entre les hommes et les femmes ? Le métier d'enseignant entraîne-t-il une distribution moins genrée des tâches parentales?

\section{Méthodologie de l'enquête}

Notre recherche s'appuie donc sur l'exploitation de l'enquête Emploi du temps de 2010, et du module " Enseignants ». Notre analyse porte sur les professeurs agrégés et certifiés de l'enseignement secondaire, les enseignants de l'enseignement supérieur, les instituteurs, les professeurs des écoles, les professeurs de lycée professionnel, les maitres auxiliaires et professeurs contractuels de l'enseignement secondaire. La taille de l'échantillon ne permet toutefois pas de distinguer dans les comparaisons réalisées les enseignants du premier ou du second degré, ni de tenir compte de la matière qu'ils enseignent, même si ces deux caractéristiques sont susceptibles d'avoir une influence sur leur disponibilité temporelle 5 .

\footnotetext{
4. Cette enquête propose en effet un sur-échantillon d'enseignants du primaire et du secondaire, basé sur 1094 adresses, avec un taux de sondage moyen de 1/633 (pour les logements issus de la base d'enseignants). Cela permet notamment d'appréhender le temps de travail des enseignants du primaire et du secondaire.
} 
Les parents enseignants sont comparés à des parents diplômés de l'enseignement supérieur, en prenant comme critère la possession d'au moins une licence pour cette catégorie. Ce niveau a été choisi car il correspond à la situation la plus fréquente chez les enseignants ${ }^{6}$.

Compte tenu de notre questionnement, le champ de notre recherche concerne par ailleurs des individus vivant dans un ménage comprenant au moins un enfant de moins de 18 ans, en couple ou à la tête d'une famille monoparentale, dont le statut est « en emploi », ces individus étant la personne de référence ou son conjoint ${ }^{7}$. Notre échantillon est constitué de 552 enseignants (357 femmes et 197 hommes) et de 766 diplômés de l'enseignement supérieur (393 femmes et 373 hommes).

Notre intérêt se portant sur l'usage du temps parental ${ }^{8}$, nous avons choisi d'étudier le temps total consacré aux enfants, mais aussi d'analyser séparément, au sein de ce temps, le temps consacré aux jeux et à l'instruction, et le temps consacré aux activités de soins et d'accompagnement (cf. encadré 2 pour une description des activités concernées).

Le temps total présente de l'intérêt dans le sens où, comme l'écrit Da-Costa Lasne (2012) qui a cherché à identifier les spécificités des pratiques éducatives parentales des enseignants, "l'accompagnement scolaire parental ne s'exerce pas sporadiquement par des activités ciblées, mais continument au travers des pratiques les plus quotidiennes » (p. 304). Nous distinguons

5. Le nombre moyen d'heures travaillées est de $42 \mathrm{~h}$ par semaine pour les disciplines littéraires, 39 h55 pour les disciplines scientifiques et 38h45 pour les langues vivantes (MENERS, 2011, p. 305).

6. Les réformes Jospin de 1989-1991 ont créé les Instituts universitaires de formation des maîtres (IUFM) et unifié à la licence le niveau de recrutement de la plupart des enseignants. Depuis 2008, ce recrutement se fait au niveau Master.

7. Dans l'échantillon retenu, les familles recomposées ont été conservées.

8. L'enquête Emploi du temps permet de différencier des activités codées comme principales d'activités éventuellement réalisées en même temps, codées alors comme secondaires. Dans l'article, en l'absence de précisions, les activités étudiées sont les activités principales.

\section{Encadré 1}

\section{LES CARACTÉRISTIQUES DES DEUX POPULATIONS ÉTUDIÉES}

\section{(cf. tableau A en annexe)}

Les femmes sont surreprésentées dans la profession enseignante : $70 \%$ contre $30 \%$, alors que l'on a un relatif équilibre chez les diplômés non enseignants. Cette répartition correspond à ce que l'on connaît du monde enseignant : en janvier 2011, il y avait $66 \%$ de femmes dans l'enseignement de secteur public et $74 \%$ dans le secteur privé (MENERS, 2011, p. 283).

Les diplômés non enseignants ont plus souvent moins de 35 ans que les enseignants ; ces derniers ont plus souvent entre 35 et 44 ans. Pour la dernière catégorie ( 45 ans et plus »), la répartition est la même. Cette répartition est conforme à l'évolution de la structure par âge de la population enseignante décrite par Farges (2010), évolution due notamment à une forte vague de recrutement au début des années 90 . Cette structure par âge a une répercussion sur le nombre et l'âge des enfants : les enseignants ont plus souvent que les diplômés non enseignants seulement des enfants âgés de plus de 6 ans. Autrement dit, ces derniers ont plus souvent que les enseignants un seul enfant âgé de 0 à 6 ans.

Compte tenu de la définition de notre population d'étude et des niveaux de recrutement des enseignants, les diplômés non enseignants ont plus souvent un diplôme de $3^{e}$ cycle ou de grandes écoles, que les enseignants.
En ce qui concerne la configuration familiale, il y a autant de familles monoparentales dans les deux cas. $\mathrm{Si}$ on exclut les familles monoparentales, $34 \%$ des enseignants sont en couple avec un enseignant contre $10 \%$ des diplômés non enseignants : on retrouve l'importante homogamie professionnelle du corps enseignant décrite par Farges (2010). 50 \% des hommes enseignants sont en couple avec une enseignante contre $14 \%$ des hommes diplômés non enseignants. $27 \%$ des femmes enseignantes sont en couple avec un enseignant, contre $6 \%$ des femmes diplômées non-enseignantes. Par ailleurs, l'homogamie scolaire est forte, chez les enseignants et les non-enseignants.

Les enseignants sont légèrement plus souvent issus du monde enseignant puisque 19,5\% (respectivement $17 \%$ des enseignants du premier degré et $23 \%$ des enseignants du second degré) ont au moins un parent enseignant. C'est le cas de $16 \%$ des diplômés non enseignants.

Enfin, les enseignants sont beaucoup mieux répartis sur le territoire que ne le sont les diplômés nonenseignants. Cet aspect est lié aux modalités d'affectation des enseignants des premier et second degrés, et à leur répartition sur tout le territoire, ainsi qu'à la concentration des emplois de cadres, et par-là des diplômés, dans les zones urbaines et plus précisément dans les capitales régionales (Caro et Hillau, 1997). 
par ailleurs le temps consacré aux soins et à l'accompagnement et le temps consacré aux jeux et à l'instruction pour deux raisons principales : tout d'abord, nous formulons l'hypothèse que l'usage du temps parental des enseignants peut être différent de celui des autres parents concernant des activités proches du monde scolaire, cet usage découlant de leurs stratégies éducatives (Da-Costa Lasne, 2012) ${ }^{9}$. Ensuite, il nous paraît important de distinguer ces deux temps pour arriver à cerner finement les différences entre les hommes et les femmes. En effet, il est établi que ces dernières consacrent beaucoup plus de temps que les hommes aux tâches ménagères (Ricroch et Roumier, 2011) et aux tâches parentales. De plus, lorsque les hommes y consacrent du temps, c'est plus souvent dans le cadre de loisirs partagés que pour des activités « plus proches du maternage », ces activités étant prises en charge essentiellement par les femmes (Algava, 2002 ; Brugeilles et Sebille, 2009).

Nous nous intéressons alors aux comportements de nos deux populations en les distinguant selon leur sexe, et à la répartition des tâches parentales, notamment quand les deux conjoints sont enseignants. Les hommes enseignants s'impliquent-ils davantage que les autres hommes diplômés dans des tâches parentales habituellement dévolues aux mères ? ${ }^{9}$

Le temps consacré aux enfants (et, au-delà, le type de temps) dépend du sexe des parents et plus généralement de leurs caractéristiques sociodémographiques. C'est pourquoi nous nous sommes intéressées à l'âge, au niveau de diplôme des individus, à celui de leurs parents, au milieu d'origine et enfin, à l'environnement géographique.

Mais ce temps dépend également des caractéristiques de la famille, comme l'ont montré Brugeilles et Sebille (2009) ; nous avons alors

\footnotetext{
9. Si notre sujet inviterait à s'intéresser plus spécifiquement au temps consacré à l'accompagnement scolaire, cela n'est pas possible pour deux raisons : cela nous obligerait à restreindre notre population aux personnes avec des enfants de plus de 6 ans, ce que ne permettent pas les effectifs; les activités prises en compte dans les jeux et l'instruction ne sont pas suffisamment pratiquées sur une journée décrite pour être étudiées séparément.
}

Encadré 2

\section{LES ACTIVITÉS PRISES EN COMPTE}

Les activités décrites par les individus dans les carnets journaliers sont regroupées à partir de la nomenclature d'activités de l'enquête EDT 2010-2011.

Le temps consacré aux soins des enfants et aux trajets d'accompagnement, par jour, est la somme des durées des activités codées sous les appellations suivantes :

- S'occuper d'enfants de son ménage (hors soins médicaux)

- Accompagner un enfant de son ménage, l'attendre (hors trajets)

- Soins médicaux aux enfants de son ménage, à domicile

- Autres : bisous, câlins, gronderies... à un enfant de son ménage

- Trajets liés aux enfants

Le temps consacré aux jeux et à l'instruction des enfants, par jour, est la somme des durées des activités codées sous les appellations suivantes :

- Surveillance des devoirs et leçons

- Conversations, lectures non scolaires

- Jeux et activités à domicile

- Jeux et activités hors du domicile
Le temps total par jour consacré aux enfants est la somme du temps consacré aux soins et aux trajets d'accompagnement, et du temps consacré aux jeux et à l'instruction des enfants.

Le « noyau dur » du travail domestique, par jour, est la somme des durées des activités codées sous les appellations suivantes :

- Cuisine : préparation et cuisson des aliments, épluchage

- Lavage de la vaisselle et rangement de la vaisselle, débarrasser la table

- Mettre la table, servir le repas

- Rangement des courses, chargement et déchargement de la voiture

- Rangement et nettoyage extérieur

- Ménage et rangement (intérieur de la maison)

- Lavage du linge (y c. le trier, le mettre dans / le sortir de la machine à laver, l'étendre)

- Repassage

- Couture, tricot, crochet, cirage et lavage des chaussures

- Rangement des vêtements, préparer son sac, sa valise 
pris en compte l'âge et le nombre d'enfant(s) ${ }^{10}$, ainsi que le type de ménage d'appartenance (en distinguant la situation " en couple avec un(e) enseignant(e) »).

La population enseignante présentant certaines spécificités par rapport à une population d'individus non enseignants possédant au moins une licence, nous exposons dans l'encadré 1 les principales différences entre ces deux populations.

Dans notre article, nous comparons les temps moyens consacrés aux enfants et les taux de participation aux activités parentales chez les hommes et les femmes de ces deux groupes. Cette comparaison est complétée par des analyses de ces temps toutes choses égales par ailleurs, analyses d'autant plus nécessaires que la population d'enseignants possède certaines particularités (cf. encadré 1). D'éventuels phénomènes de compensation sont également recherchés. Les non-enseignants compensent-ils leur éventuel moindre investissement parental les jours travaillés, par un sur-investissement les jours non travaillés (cf. encadré 3 pour une définition) ? ${ }^{11}$ Ont-ils tendance à cumuler davantage leurs activités ? Autrement dit, les différences constatées sur les temps déclarés en activités principales subsistent-elles lorsque les activités secondaires sont ajoutées ? Ensuite, avec l'étude des enseignants en couple, nous regardons si les enseignant(e)s dont le(la) conjoint(e) ne l'est pas se spécialisent, ou non, dans la prise en charge des enfants.

10. Le sexe des enfants ne peut pas être pris en compte, les carnets sur lesquels les individus décrivent leurs activités ne précisant pas à quel enfant du ménage l'adulte consacre du temps, ni le sexe de celui-ci.

11. Il aurait pu être intéressant d'étudier des phénomènes de compensation au sein de chaque couple. Cela n'a pas été possible car le choix de faire la distinction entre les jours travaillés et non travaillés ne nous permettait pas, pour des raisons d'effectifs, d'apparier également les membres des couples.

\section{Encadré 3}

\section{LES ANALYSES RÉALISÉES TOUTES CHOSES ÉGALES PAR AILLEURS}

Les durées ont fait l'objet d'analyses par sexe en utilisant des modèles Tobit simple (cf. ci-dessous), ces modèles permettant de prendre en compte le fait que certaines durées sont nulles. La procédure QLIM de Sas a été utilisée à cet effet.

\section{Les variables prises en compte}

Les analyses effectuées toutes choses égales par ailleurs sur le temps total consacré aux enfants, en considérant séparément l'effet du sexe, de la profession (enseignant ou non) et du type de jour décrit (travaillé ou non) ont mis en évidence une interaction sexe, profession, et type de journée. Ceci nous a amenées à construire une variable combinant le sexe, la profession et le fait que le jour décrit soit travaillé ou non. Toutes les analyses sur les durées des activités ont été réalisées avec cette variable, les autres variables prises en compte dans le modèle restant inchangées. Ceci permet de comparer les enseignants et les diplômés non enseignants selon leur sexe et le type de jour considéré, tout en ayant un effectif suffisant pour avoir la possibilité de mettre en évidence des éventuelles différences. Les tableaux donnés dans le corps du texte et en annexe ont pour catégorie de référence pour cette variable combinée, successivement « femme non-enseignante une journée travaillée » et « homme non-enseignant une journée travaillée ", mais les analyses ont été réalisées en faisant varier la catégorie de référence en envisageant toutes les combinaisons de ces trois variables. Les tableaux prenant en compte des combinaisons non présentées ici sont disponibles auprès des auteures.
Ainsi, pour comparer les femmes entre elles, un jour non travaillé, la référence prise est « femme diplômée non-enseignante un jour non travaillé ". Pour comparer les hommes entre eux un jour travaillé et un jour non travaillé, on prend successivement comme référence " homme diplômé non-enseignant un jour travaillé » et " homme diplômé non-enseignant un jour non travaillé ". En outre, la construction de cette variable offre la possibilité de distinguer ce qui se passe une journée travaillée et une journée non travaillée, alors que les pondérations fournies par l'Insee n'ont pas été prévues pour permettre d'analyser séparément ces deux journées. En effet, les pondérations des carnets ont été effectuées sur l'ensemble des carnets, en tenant compte du fait que toutes les personnes ayant répondu à l'enquête n'avaient pas à répondre au même nombre de carnets. Les enseignants du module "Enseignants » devaient remplir deux carnets journaliers alors que les personnes répondant au module "Décisions dans les couples " en avaient un seul.

La personne enquêtée devait préciser si la journée qu'elle décrivait était une journée normale de travail ou non. Cette variable ayant été mal renseignée, une variable « journée travaillée » a été construite. Une journée lors de laquelle au moins deux heures de travail avaient été signalées dans le carnet a été considérée comme une journée travaillée, ce seuil de deux heures donnant pour les enseignants une durée moyenne de travail égale à $7 \mathrm{~h} 10$, valeur proche de celle trouvée par Chenu (2002). 
Après avoir étudié le temps consacré aux enfants les jours décrits dans les carnets de l'enquête, nous nous intéressons à la disponibilité des parents le soir et le mercredi. Qui prend habituellement en charge les enfants à ce moment-là ? Nous vérifions si l'hypothèse d'une meilleure synchronisation du temps familial avec le temps scolaire est corroborée

Encadré 3 (suite)

Les autres variables sélectionnées dans les analyses toutes choses égales par ailleurs, afin de comparer les enseignants aux diplômés non enseignants, prennent en compte les différences observées sur les caractéristiques des deux populations étudiées (cf. tableau $A$ en annexe) :

- Le type de ménage, avec trois modalités : " famille monoparentale "; " en couple avec enfants et le conjoint est enseignant "; " en couple avec enfants et le conjoint est non enseignant ». Nous avons considéré uniquement ces trois situations, sans tenir compte d'autres caractéristiques du conjoint, afin de ne pas exclure de l'analyse les familles monoparentales.

- Une variable tenant compte simultanément du nombre et de l'âge des enfants, avec cinq modalités : " un seul enfant âgé de 0 à 6 ans "; « deux enfants et plus (au moins deux ont de 0 à 6 ans); " deux enfants et plus (dont un âgé de 0 à 6 ans) »; « deux enfants et plus âgés de plus de 6 ans "; " un seul enfant âgé de plus de 6 ans ".

- L'âge en trois classes : "moins de 35 ans "; " de 35 ans à 44 ans »; " 45 ans et plus ».

- Le fait d'avoir été élevé par une mère enseignante ou non.

- Le fait d'avoir été élevé par un père enseignant ou non.

Pour ces deux dernières variables, un recodage a été réalisé à partir de la profession des parents qui était donnée sous la forme d'une question ouverte.

- Le type de commune de résidence, avec trois modalités : " commune rurale ou ville de moins de 20000 habitants "; « ville de 20000 habitants à moins de 200000 habitants "; " ville de 200000 habitants et plus ".

Les mêmes paramètres ont été pris en compte dans la régression logistique visant à étudier la probabilité de faire partie de ceux qui consacrent le plus de temps aux enfants (cf. tableaux $\mathrm{G}$ et $\mathrm{H}$ en annexe).

Enfin, les analyses réalisées séparément sur les femmes enseignantes en couple et sur les hommes enseignants en couple prennent en compte les variables "nombre et âge des enfants ", " classes d'âge ", « père enseignant », « mère enseignante » décrites ci-dessus. À ces variables ont été ajoutées une variable tenant compte de manière simultanée de la profession et du diplôme du (de la) conjoint(e) (trois cas sont distingués : " en couple avec un(e) non-enseignant(e) ayant un diplôme inférieur ou égal au baccalauréat "; " en couple avec un(e) enseignant(e) "; " en couple avec un(e) non-enseignant(e) ayant un diplôme de l'enseignement supérieur ») et une variable précisant si la journée est travaillée ou non.

Les modèles utilisés sur les durées des activités

Les durées consacrées aux activités ont été analysées par des modèles Tobit simple, ces modèles permettant de prendre en compte le fait que la durée puisse être égale à zéro en l'absence de participation aux activités étudiées.

Le modèle Tobit simple est le suivant :

Soit $Y_{i}^{*}$ une variable latente. Cette variable s'exprime de la manière suivante :

$Y_{i}^{*}=\beta X_{i}+\varepsilon_{i}$ avec $\varepsilon_{i} \sim n\left(0, \sigma^{2}\right)$

$Y_{i}$ est égale à $Y_{i}^{*}$ si $Y_{i}^{*}>0$ et à 0 sinon.

$X_{i}$ désigne un vecteur de paramètres explicatifs qui concernent les individus étudiés, $\beta$ est un vecteur de coefficients associés et $\varepsilon_{\mathrm{i}}$ est un résidu aléatoire distribué suivant une loi normale centrée de variance constante $\sigma^{2}$

$E\left[Y_{i}, X_{i}\right]=F\left(\beta X_{i} / \sigma\right)\left(\beta X_{i}+\sigma \lambda_{1}\right)$

Avec $\lambda_{i}$ est égal à $f\left(\beta X_{i} / \sigma\right) / F\left(\beta X_{i} / \sigma\right)$, et où $f(z)$ et $F(z)$ sont respectivement la fonction de densité et la fonction de répartition de la loi normale.

L'effet marginal des $X_{i}$ sur la variable latente $Y^{*}$ est égal à $\partial E\left[Y^{\prime} \backslash X\right] / \partial x_{i}=\beta_{i}$

L'effet marginal des $X_{i}$ sur la variable $Y$ est alors égal à $\partial E[Y X] / \partial x_{i}=\beta_{i} F(\beta X / \sigma)$.

Les valeurs égales à zéro étant considérées comme de vraies valeurs (c-à-d qu'une durée est nulle quand l'activité étudiée n'a pas été pratiquée) et non pas comme des valeurs non observables, on s'intéresse ici aux effets marginaux des $X_{i}$ sur la variable $Y$. II s'agit des effets présentés, dans les tableaux dans le corps de l'article et en annexe, dans la colonne « Effet marginal ».

Les modèles utilisés sur la probabilité de faire partie de ceux qui consacrent le plus de temps aux enfants

Pour étudier l'effet de la profession (enseignant ou non) sur la probabilité de faire partie de ceux qui consacrent le plus de temps aux enfants, en tenant compte des autres caractéristiques considérées comme paramètres explicatifs $V_{i}$, on a eu recours à une régression logistique. Faire partie de ce groupe a été considéré de manière dichotomique (prenant la valeur 1 si c'est le cas, et 0 dans le cas contraire). 
par cette enquête, sur les journées décrites. Le taux de présence des enseignants et des nonenseignants au domicile est particulièrement étudié entre le moment où les enfants ont terminé leur journée scolaire, et avant ou pendant le repas du soir. Pour finir, nous nous demandons si les enseignants font partie de ceux qui surinvestissent le temps parental.

\section{Le temps consacré aux enfants : les femmes enseignantes en font plus...}

\section{Le temps total}

Sur l'ensemble des hommes et des femmes étudiés ici, le temps moyen par jour (travaillé ou non) dédié aux enfants est important, et il est plus élevé pour les enseignants que pour les diplômés non enseignants. La différence existe surtout en présence d'au moins un enfant âgé de zéro à six ans dans le foyer, le temps étant alors supérieur d'une demi-heure pour les enseignants alors que la différence est de l'ordre d'une dizaine de minutes quand tous les enfants ont plus de six ans (cf. tableau 1).

L'analyse par sexe montre que ceci est principalement dû aux femmes enseignantes, qui consacrent plus de temps à leurs enfants que les diplômées non enseignantes quand elles ont des enfants jeunes (près de vingt minutes de plus dans ce cas). En revanche, le temps consacré aux enfants de plus de six ans est quasi identique pour les deux groupes de femmes (de l'ordre de une heure en moyenne). Pour les hommes, les temps consacrés aux enfants sont assez proches mais diffèrent très légèrement, au contraire, quand les enfants ont plus de six ans : les hommes enseignants passent alors en moyenne 40 minutes à s'occuper des enfants contre 28 minutes pour les hommes diplômés non enseignants. Chez les femmes, les différences sont donc un peu plus prononcées que chez les hommes, mais existent cependant uniquement quand les enfants sont jeunes, les enseignantes leur consacrant en moyenne plus de temps que les diplômées non enseignantes.

Le fait d'être enseignant ne semble pas réduire l'inégalité de la prise en charge des enfants entre les hommes et les femmes : quand il existe des enfants jeunes dans la famille, les femmes enseignantes leur consacrent en moyenne une heure de plus que les hommes enseignants ; quand tous les enfants ont plus de six ans, environ vingt minutes de plus. Chez les diplômés non enseignants, les écarts constatés entre les hommes et les femmes sont respectivement de 40 minutes et d'une demi-heure. Les différences entre les hommes et les femmes apparaissent alors même majorées pour les enseignants quand les enfants sont jeunes. Les hommes enseignants participent néanmoins plus souvent à ces tâches que les autres hommes étudiés ici. En effet, les taux de participation aux activités parentales sont toujours plus élevés (de 9 points) pour les hommes enseignants que pour les hommes diplômés non enseignants. Quand les enfants sont jeunes, une forte majorité d'hommes $(91 \%$ des enseignants et $82 \%$ des diplômés non enseignants) déclarent passer du temps à s'occuper des enfants mais les taux de participation diminuent beaucoup quand les enfants ont plus de six ans (53\% pour les enseignants contre $44 \%$ pour les diplômés non enseignants). Chez les femmes, quand il existe des enfants de zéro à six ans dans la famille, les différences entre les taux de participation sont plus réduites ( $97 \%$ pour les enseignantes contre $92 \%$ pour les non enseignantes), tandis que les taux de participation sont identiques $(65 \%)$ lorsque tous les enfants ont plus de six ans. Les durées moyennes des activités parentales de ceux qui ont déclaré du temps pour ces tâches connaissent les mêmes variations que celles qui sont observées sur l'ensemble.

\section{Les jeux et l'instruction}

Le temps consacré aux jeux et à l'instruction des enfants est également un peu plus élevé chez les enseignants que chez les diplômés non enseignants quand il existe des enfants jeunes dans la famille, mais la différence est faible (30 minutes contre 23 minutes). Quand les enfants sont plus grands, les valeurs moyennes sont quasiment identiques.

La différence constatée quand les enfants sont jeunes est en fait due aux enseignantes, qui consacrent en moyenne 10 minutes de plus à ce type d'activités que les diplômées non enseignantes. Chez les hommes, les valeurs moyennes sont plus voisines, mais contrairement aux femmes, les hommes enseignants passent quelques minutes de plus à ces activités que les hommes diplômés non enseignants quand les enfants ont plus de six ans.

Les taux de participation à des activités telles que « jeux et instruction des enfants » pourraient témoigner de la plus grande importance attachée par les enseignants aux activités éducatives, surtout quand la famille comprend 


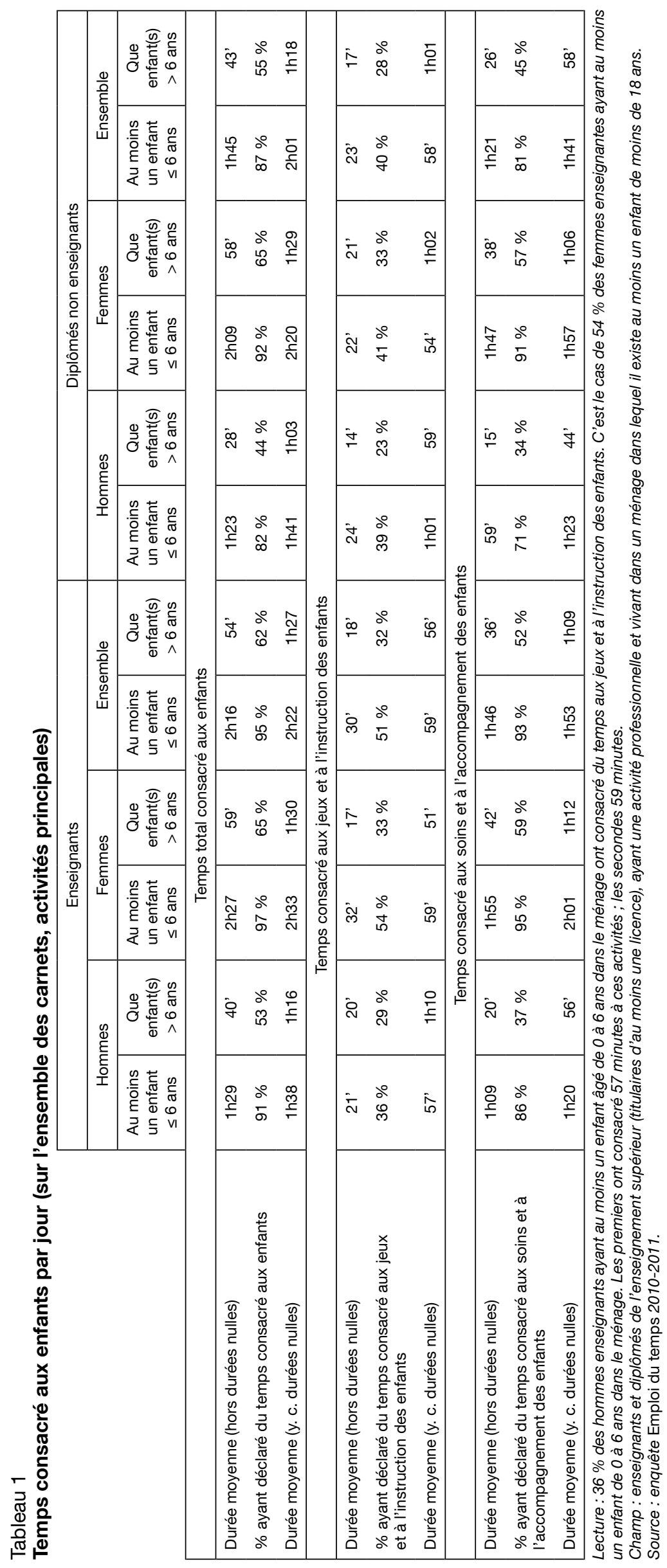


des enfants ayant moins de six ans. $51 \%$ des enseignants contre $40 \%$ des diplômés nonenseignants passent alors du temps à de telles activités, tandis que ceci concerne $32 \%$ des enseignants et $28 \%$ des non-enseignants quand tous les enfants ont plus de six ans.

Pour les taux de participation à ces activités, les différences existent principalement pour les femmes : $54 \%$ d'entre elles y consacrent du temps contre $41 \%$ des autres diplômées si la famille comporte au moins un enfant jeune. Quand les enfants sont plus âgés, ceci concerne un tiers des femmes de chacun des deux groupes. Chez les hommes, les taux de participation sont plus voisins : on note toutefois une participation un peu plus importante des enseignants que des autres diplômés quand les enfants ont plus de six ans. Les durées moyennes de ces activités, quand elles sont pratiquées, sont dans la plupart des cas de l'ordre d'une heure mais apparaissent augmentées de 10 minutes chez les hommes enseignants quand les enfants ont plus de six ans, alors qu'elles sont diminuées de la même durée pour les femmes enseignantes. À noter que pour les jeux et l'instruction, les durées moyennes de ces activités sont très voisines chez les hommes enseignants et les femmes non enseignantes.

\section{Les soins et l'accompagnement}

Les femmes enseignantes consacrent en moyenne un peu plus de temps à ces activités que les femmes diplômées non enseignantes quand les enfants sont jeunes, et déclarent plus souvent ces activités, les différences étant toutefois peu importantes $(95 \%$ des enseignantes contre $91 \%$ des non-enseignantes ont déclaré consacrer du temps aux soins et à l'accompagnement s'il existe des enfants âgés de zéro à six ans). Quand elles déclarent de telles activités, elles y consacrent une vingtaine de minutes de plus que les non-enseignantes.

Les différences constatées entre les hommes sont plus nettes, surtout en ce qui concerne les taux de participation : en présence de jeunes enfants, $86 \%$ des hommes enseignants réalisent ce type d'activités, contre $71 \%$ des hommes diplômés non enseignants. Comme dans les enquêtes citées précédemment, on observe que les activités de soins et d'accompagnement des enfants sont principalement assignées aux femmes. Ici, le temps qui y est consacré par les femmes est presque deux fois plus important que celui consacré par les hommes, et apparaît dépendre plus du sexe du parent que de sa profession.

\section{Le temps parental dépend d'abord de l'âge des enfants et de leur nombre}

Les différents types de temps consacrés aux enfants ont été comparés chez les enseignants et les non-enseignants, par des analyses toutes choses égales par ailleurs qui permettent de prendre en compte le fait que la population d'enseignants présente certaines spécificités, comparée à la population des autres diplômés du supérieur (cf. encadré 3 et tableaux 2a et $2 b$ ).

Le temps consacré aux enfants dépend en premier lieu de l'âge et du nombre des enfant(s) : en prenant comme référence un seul enfant âgé de plus de six ans, le temps total (en raison du temps consacré aux soins et à l'accompagnement) est d'autant plus augmenté que les enfants sont jeunes et nombreux. Le temps consacré aux jeux et à l'instruction est plus important dans les familles comprenant un ou deux jeunes enfants que dans celles ayant un seul enfant de plus de six ans.

L'âge des parents joue également un rôle: ceux de moins de 44 ans vouent toujours à leurs enfants plus de temps que ceux de plus de 45 ans. Cet effet est probablement un effet indirect de l'âge des enfants ${ }^{12}$. Le fait de vivre sans conjoint augmente également le temps total consacré aux enfants et aux activités de soins et d'accompagnement, mais pas le temps passé aux jeux et à l'instruction.

Par ailleurs, plus de temps est consacré aux enfants dans les grandes agglomérations qu'en zone rurale ${ }^{13}$, en raison du temps voué aux jeux et à l'instruction (augmenté également dans les villes de 20000 à 200000 habitants).

Avoir été élevé par une mère enseignante augmente un peu le temps consacré aux enfants, variation qui s'explique par un temps de soins et d'accompagnement plus important ; ceci pourrait être la marque d'une transmission d'un certain modèle maternel. Henri-Panabière (2010), dans son enquête sur les " méshéritiers », a d'ailleurs bien montré l'importance

\footnotetext{
12. Sachant que la distinction "plus de 6 ans / 0 à 6 ans » opérée - pour des raisons d'effectifs - est assez sommaire.

13. Il a été vérifié que ceci ne s'explique pas par des trajets liés aux enfants, ni par la possession ou non d'un jardin (attribut plus fréquent en zone rurale). Une hypothèse pourrait être une aide moins importante de la famille au cours de la journée, dans les grandes agglomérations. En effet, au cours du mois précédent, dans notre population, $37 \%$ de ceux qui sont en zone rurale ou dans les villes de moins de 20000 habitants ont été aidés au moins une fois par de la famille; c'est le cas de $42 \%$ de ceux qui sont dans les villes de 20000 à 200000 habitants et seulement $26 \%$ des habitants des grandes agglomérations.
} 
Tableau 2a

Temps consacré aux enfants

Résultats des estimations des modèles Tobit

(Référence : Femme diplômée non enseignante un jour travaillé)

\begin{tabular}{|c|c|c|c|c|c|c|}
\hline Coefficients, effets marginaux et valeurs de $p$ & \multicolumn{2}{|c|}{ Temps total } & \multicolumn{2}{|c|}{$\begin{array}{l}\text { Temps consacré } \\
\text { aux jeux } \\
\text { et à l'instruction }\end{array}$} & \multicolumn{2}{|c|}{$\begin{array}{c}\text { Temps consacré } \\
\text { aux soins } \\
\text { et à l'accompagnement }\end{array}$} \\
\hline$\%$ concernés par l'activité & \multicolumn{2}{|c|}{73} & \multicolumn{2}{|c|}{35} & \multicolumn{2}{|c|}{65} \\
\hline \multirow[t]{2}{*}{ Moyennes sur l'ensemble } & \multicolumn{2}{|c|}{$78^{\prime}$} & \multicolumn{2}{|c|}{$21^{\prime}$} & \multicolumn{2}{|c|}{$57^{\prime}$} \\
\hline & Coefficient & $\begin{array}{c}\text { Effet } \\
\text { marginal }\end{array}$ & Coefficient & $\begin{array}{c}\text { Effet } \\
\text { marginal }\end{array}$ & Coefficient & $\begin{array}{c}\text { Effet } \\
\text { marginal }\end{array}$ \\
\hline \multicolumn{7}{|c|}{ Sexe et profession et type de journée } \\
\hline Femme enseignante un jour travaillé & $+28^{\star \star \star}$ & +19 & +26 *** & +9 & $+19^{*}$ & +12 \\
\hline Femme enseignante un jour non travaillé & $+37^{\star \star \star}$ & +25 & $+37^{\star \star \star}$ & +13 & $+19^{*}$ & +12 \\
\hline Femme diplômée non enseignante un jour travaillé & Réf. & & Réf. & & Réf. & \\
\hline $\begin{array}{l}\text { Femme diplômée non enseignante un jour non } \\
\text { travaillé }\end{array}$ & $+52^{\star \star \star}$ & +36 & $+35^{\star \star \star}$ & +12 & $+34^{\star * \star}$ & \\
\hline Homme enseignant un jour travaillé & -4 & & $+38^{\star \star}$ & +14 & $-34^{\star *}$ & -21 \\
\hline Homme enseignant un jour non travaillé & -33 & & -1 & & $-40^{\star *}$ & -25 \\
\hline Homme diplômé non enseignant un jour travaillé & $-42^{\star \star \star}$ & -29 & -4 & & $-48^{\star \star \star}$ & -30 \\
\hline Homme diplômé non enseignant un jour non travaillé & +1 & & $+33^{\star \star \star}$ & +12 & $-25^{\star \star \star}$ & -16 \\
\hline \multicolumn{7}{|c|}{ Type de ménage } \\
\hline $\begin{array}{l}\text { Famille monoparentale } \\
\text { En couple avec enfant(s) } \\
\text { Le conjoint n'est pas enseignant }\end{array}$ & $\begin{array}{c}+25^{\star \star \star} \\
\text { Réf. }\end{array}$ & +17 & $\begin{array}{l}-4 \\
\text { Réf. }\end{array}$ & & $\begin{array}{c}+29^{\star \star \star} \\
\text { Réf. }\end{array}$ & +18 \\
\hline $\begin{array}{l}\text { En couple avec enfant(s) } \\
\text { Le conjoint est enseignant }\end{array}$ & +7 & & $-17^{\star \star}$ & -6 & +14 & +9 \\
\hline \multicolumn{7}{|c|}{ Nombre et âge des enfants } \\
\hline Un seul enfant d'âge $\leq 6$ ans & $+112^{\star \star \star}$ & +78 & $+17^{\star \star}$ & +6 & $+118^{\star \star \star}$ & +73 \\
\hline Deux enfants et plus (au moins deux d'âge $\leq 6$ ans) & $+164^{\star \star \star}$ & +114 & $+35^{\star \star \star}$ & +12 & $+162^{\star \star \star}$ & +101 \\
\hline Deux enfants et plus (dont un d'âge $\leq 6$ ans) & $+91^{\star \star \star}$ & +63 & +3 & & $+103^{\star \star \star}$ & +64 \\
\hline Deux enfants et plus d'âge $>6$ ans & $+50^{\star \star \star}$ & +35 & +6 & & $+54^{\star \star \star}$ & +33 \\
\hline $\begin{array}{l}\text { Un seul enfant âgé de plus de } 6 \text { ans (et de moins } \\
\text { de } 18 \text { ans) }\end{array}$ & Réf. & & Réf. & & Réf. & \\
\hline \multicolumn{7}{|c|}{ Classes d'âge } \\
\hline$<35$ ans & $+28^{\star \star \star}$ & +20 & $+35^{\star \star \star}$ & +12 & $+18^{\star \star}$ & +11 \\
\hline 35 à 44 ans & $+31^{\star \star \star}$ & +22 & $+38^{\star \star \star}$ & +13 & $+20^{\star *}$ & +13 \\
\hline 45 ans et + & Réf. & & Réf. & & Réf. & \\
\hline \multicolumn{7}{|c|}{ Parent(s) enseignant(s) } \\
\hline Mère enseignante & $+13^{*}$ & +9 & +5 & & $+16^{\star \star}$ & +10 \\
\hline Mère non enseignante & Réf. & & Réf. & & Réf. & \\
\hline Père enseignant & -15 & & +1 & & $-20^{\star \star}$ & -13 \\
\hline Père non enseignant & Réf. & & Réf. & & Réf. & \\
\hline \multicolumn{7}{|c|}{ Type de commune } \\
\hline Rurale à moins de 20000 hab. & Réf. & & Réf. & & Réf. & \\
\hline 20000 hab. à moins de 200000 hab. & +12 & & $+19^{\star \star \star}$ & +7 & +6 & \\
\hline 200000 hab. et + & $+14^{\star *}$ & +10 & $+30^{\star \star \star}$ & +10 & -2 & \\
\hline
\end{tabular}

Note : ${ }^{* * \star}$ significatif à $1 \% ; *$ à $5 \%$; * à $10 \% ;-$ : non significatif ; Réf. : catégorie de référence. Les effets marginaux sont précisés seulement quand le coefficient est significatif.

Lecture : un jour travaillé, les femmes enseignantes consacrent 25 minutes de plus aux enfants que les femmes diplômées non enseignantes.

Champ : enseignants et diplômés de l'enseignement supérieur (titulaires d'au moins une licence), ayant une activité professionnelle et vivant dans un ménage dans lequel il existe au moins un enfant de moins de 18 ans.

Source : enquête Emploi du temps 2010-2011. 
Tableau 2b

Temps consacré aux enfants

Résultats des estimations des modèles Tobit

(Référence : Homme diplômé non enseignant un jour travaillé)

\begin{tabular}{|c|c|c|c|c|c|c|}
\hline Coefficients, effets marginaux et valeurs de $p$ & \multicolumn{2}{|c|}{ Temps total } & \multicolumn{2}{|c|}{$\begin{array}{l}\text { Temps consacré aux } \\
\text { jeux et à l'instruction }\end{array}$} & \multicolumn{2}{|c|}{$\begin{array}{c}\text { Temps consacré aux } \\
\text { soins et à l'accompa- } \\
\text { gnement }\end{array}$} \\
\hline$\%$ concernés par l'activité & \multicolumn{2}{|c|}{73} & \multicolumn{2}{|c|}{35} & \multicolumn{2}{|c|}{65} \\
\hline \multirow[t]{2}{*}{ Moyennes sur l'ensemble } & \multicolumn{2}{|c|}{78} & \multicolumn{2}{|c|}{$21^{\prime}$} & \multicolumn{2}{|c|}{57} \\
\hline & Coefficient & $\begin{array}{c}\text { Effet } \\
\text { marginal }\end{array}$ & Coefficient & $\begin{array}{c}\text { Effet } \\
\text { marginal }\end{array}$ & Coefficient & $\begin{array}{c}\text { Effet } \\
\text { marginal }\end{array}$ \\
\hline \multicolumn{7}{|c|}{ Sexe et profession et type de journée } \\
\hline Femme enseignante un jour travaillé & $+69^{\star \star \star}$ & +48 & $+30^{\star \star \star}$ & +11 & $+67^{\star \star \star}$ & +41 \\
\hline Femme enseignante un jour non travaillé & $+79^{\star \star \star}$ & +55 & $+42^{\star \star \star}$ & +15 & $+67^{\star \star \star}$ & +42 \\
\hline Femme diplômée non enseignante un jour travaillé & $+42^{\star \star \star}$ & +29 & +4 & & $+48^{\star \star \star}$ & +30 \\
\hline Femme diplômée non enseignante un jour non travaillé & $+94^{\star \star \star}$ & +65 & $+40^{\star \star \star}$ & +14 & $+82^{\star \star \star}$ & +51 \\
\hline Homme enseignant un jour travaillé & $+38^{\star \star}$ & +26 & $+43^{\star \star \star}$ & +15 & +13 & \\
\hline Homme enseignant un jour non travaillé & +9 & & +4 & & +8 & \\
\hline Homme diplômé non enseignant un jour travaillé & Réf. & & Réf. & & Réf. & \\
\hline Homme diplômé non enseignant un jour non travaillé & $+43^{\star \star \star}$ & +30 & $+38^{\star \star \star}$ & +13 & $+23^{\star \star \star}$ & +14 \\
\hline \multicolumn{7}{|c|}{ Type de ménage } \\
\hline Famille monoparentale & $+25^{\star \star \star}$ & +17 & -4 & & $+29^{\star \star \star}$ & +18 \\
\hline $\begin{array}{l}\text { En couple avec enfant(s) } \\
\text { Le conjoint n'est pas enseignant }\end{array}$ & Réf. & & Réf. & & Réf. & \\
\hline $\begin{array}{l}\text { En couple avec enfant(s) } \\
\text { Le conjoint est enseignant }\end{array}$ & +7 & & $-17^{\star \star}$ & -6 & +14 & +9 \\
\hline \multicolumn{7}{|c|}{ Nombre et âge des enfants } \\
\hline Un seul enfant d'âge $\leq 6$ ans & $+112^{\star \star \star}$ & +78 & $+17^{\star *}$ & +6 & $+118^{\star \star \star}$ & +73 \\
\hline Deux enfants et plus (au moins deux d'âge $\leq 6$ ans) & $+164^{\star \star \star}$ & +114 & $+35^{\star \star \star}$ & +12 & $+162^{\star \star \star}$ & +101 \\
\hline Deux enfants et plus (dont un d'âge $\leq 6$ ans) & $+91^{\star \star \star}$ & +63 & +3 & & $+103^{\star \star \star}$ & +64 \\
\hline Deux enfants et plus d'âge $>6$ ans & $+50^{\star \star \star}$ & +35 & +6 & & $+54^{\star \star \star}$ & +33 \\
\hline $\begin{array}{l}\text { Un seul enfant âgé de plus de } 6 \text { ans (et de moins } \\
\text { de } 18 \text { ans) }\end{array}$ & Réf. & & Réf. & & Réf. & \\
\hline \multicolumn{7}{|c|}{ Classes d'âge } \\
\hline$<35$ ans & $+28^{\star \star \star}$ & +20 & $+35^{\star \star \star}$ & +12 & $+18^{\star \star}$ & +11 \\
\hline 35 à 44 ans & $+31^{\star * \star}$ & +22 & $+38^{\star \star \star}$ & +13 & $+20^{\star \star}$ & +13 \\
\hline 45 ans et + & Réf. & & Réf. & & Réf. & \\
\hline \multicolumn{7}{|c|}{ Parent(s) enseignant(s) } \\
\hline Mère enseignante & $+13^{*}$ & +9 & +5 & & $+16^{\star \star}$ & +10 \\
\hline Mère non enseignante & Réf. & & Réf. & & Réf. & \\
\hline Père enseignant & -15 & & +1 & & $-20^{\star *}$ & -13 \\
\hline Père non enseignant & Réf. & & Réf. & & Réf. & \\
\hline \multicolumn{7}{|c|}{ Type de commune } \\
\hline Rurale à moins de 20000 hab. & Réf. & & Réf. & & Réf. & \\
\hline 20000 hab. à moins de 200000 hab. & +12 & & $+19^{\star \star \star}$ & +7 & +6 & \\
\hline 200000 hab. et + & $+14^{\star *}$ & +10 & $+30^{\star \star \star}$ & +10 & -2 & \\
\hline
\end{tabular}

Note : ${ }^{* *}$ significatif à $1 \% ;{ }^{* *}$ à $5 \%$; ${ }^{*}$ à $10 \%$; - : non significatif ; Réf. : catégorie de référence. Les effets marginaux sont précisés seulement quand le coefficient est significatif.

Lecture : un jour travaillé, les hommes enseignants consacrent 26 minutes de plus aux enfants que les hommes diplômés non enseignants.

Champ : enseignants et diplômés de l'enseignement supérieur (titulaires d'au moins une licence), ayant une activité professionnelle et vivant dans un ménage dans lequel il existe au moins un enfant de moins de 18 ans.

Source : enquête Emploi du temps 2010-2011. 
de la transmission de modèles éducatifs dans la mise en place de trajectoires scolaires d'échec ou de réussite.

Enfin, il semble exister, pour le temps voué aux jeux et à l'instruction, une forme de complémentarité dans les couples car il est un tout petit peu diminué quand le conjoint est enseignant. Gouyon et Guérin (2006) avaient constaté le même type de phénomène en ce qui concerne spécifiquement le suivi de la scolarité : «lorsque l'un des parents est enseignant, le conjoint passe moins de temps que la moyenne à aider ses enfants » (p. 70). Tout se passe comme si le fait d'être enseignant témoignait de compétences particulières, mobilisables pour ces activités. Cette hypothèse semble renforcée par le fait que, dans ce cas, on ne note pas de variation significative pour le temps consacré aux soins et à l'accompagnement.

Après avoir étudié l'effet de ces facteurs, nous nous sommes penchées plus précisément sur l'effet de la combinaison des variables sexe, profession et type de journée.

\section{L'effet « parent enseignant » au prisme des temps parentaux}

Si l'âge et le nombre des enfants apparaissent comme des facteurs prépondérants, ces analyses toutes choses égales par ailleurs mettent également en lumière que les enseignants consacrent plus de temps à s'occuper des enfants, mais que cette activité reste essentiellement genrée.

\section{Les enseignants en font plus les jours travaillés}

La comparaison entre les femmes enseignantes et les femmes diplômées non enseignantes (cf. tableau 2a) montre que lors d'un jour travaillé, les premières passent plus de temps à s'occuper de leurs enfants que les secondes (environ 20 minutes). L'analyse par type de temps fait apparaître que c'est vrai aussi bien pour le temps consacré aux jeux et à l'instruction, que pour celui consacré aux autres activités liées aux enfants.

Un jour non travaillé, le temps total consacré aux enfants augmente (de manière significative chez les non-enseignantes - d'un peu plus d'une demi-heure - mais pas chez les enseignantes). Malgré cela, ce type de temps n'est pas différent pour les enseignantes et non-enseignantes. Pour le temps consacré aux jeux et à l'instruction ainsi que pour le temps consacré aux autres activités, les mêmes phénomènes s'observent : le différentiel de temps existant entre les deux groupes n'est pas compensé.

Quant aux hommes enseignants, ils passent près d'une demi-heure de plus à s'occuper des enfants que les hommes diplômés non enseignants un jour travaillé (cf. tableau 2b). Mais l'analyse par type de temps montre que c'est dû au temps consacré aux jeux et à l'instruction, car le temps consacré aux autres activités ne diffère pas.

Un jour non travaillé, le temps total consacré aux enfants augmente chez les hommes diplômés non enseignants de manière significative, alors que ce n'est pas le cas chez les hommes enseignants, pour qui il aurait même tendance à baisser. Le temps consacré aux enfants par les hommes diplômés non enseignants ne diffère cependant pas significativement de celui des hommes enseignants. Le même phénomène est constaté pour les temps voués aux jeux et à l'instruction des enfants, ainsi qu'aux activités de soins et d'accompagnement, qui augmentent chez les diplômés non enseignants de manière significative, en l'absence de travail, alors que ce n'est pas le cas chez les enseignants.

\section{Les hommes enseignants : encore un effort...}

Les hommes et les femmes retenus dans notre analyse ont tous fait des études supérieures et exercent une activité professionnelle, cette dernière étant similaire dans la population des enseignants. Cette étude donne donc l'opportunité d'étudier dans quelle mesure les inégalités de prise en charge des enfants par les femmes et les hommes subsistent quand les conditions sont a priori favorables à plus d'égalité entre les sexes.

L'analyse du temps total montre que les hommes enseignants passent moins de temps à s'occuper de leurs enfants que les femmes enseignantes ; cette différence (de l'ordre d'une vingtaine de minutes) est encore plus prononcée un jour de repos (de l'ordre d'une cinquantaine de minutes).

Si l'on s'intéresse au type de temps, il s'avère qu'un jour travaillé, le temps qu'ils consacrent aux jeux et à l'instruction n'est pas différent de celui des femmes enseignantes, qu'elles travaillent ou non. En revanche lorsqu'ils ne travaillent pas, ils consacrent moins de temps à ces activités que ne le font les femmes enseignantes dans la même situation. Les hommes 
enseignants compenseraient donc leur investissement dans ce type de temps auprès de leurs enfants les jours travaillés, par une mise en retrait les jours non travaillés, ce qui n'est pas le cas des enseignantes. Enfin, ils consacrent moins de temps aux activités de soins et d'accompagnement que les femmes enseignantes, un jour de travail ou non.

L'analyse du temps total montre que les hommes diplômés non enseignants consacrent moins de temps aux enfants que les femmes diplômées non enseignantes (de l'ordre d'une demi-heure), que l'on soit un jour travaillé pour les deux, ou un jour non travaillé pour les deux. Le temps qu'ils consacrent aux enfants, toutes choses égales par ailleurs, est équivalent à celui des femmes diplômées non enseignantes, seulement quand elles travaillent, et eux non.

Si l'on s'intéresse au type de temps, le temps consacré aux jeux et à l'instruction est du même ordre pour les diplômés non enseignants des deux sexes, pour un type de jour donné (travaillé ou non). En revanche, le temps consacré aux activités de soins et d'accompagnement est toujours plus élevé pour les femmes que pour les hommes.

Nos résultats montrent que, lorsque leur disponibilité temporelle est a priori similaire, les femmes consacrent plus de temps aux enfants que les hommes. Que se passe-t-il lorsque les hommes sont censés avoir plus de temps disponible que les femmes ? Comparer les hommes enseignants aux femmes non enseignantes un jour de travail donne des éléments de réponse à cette question.

Si les hommes enseignants consacrent effectivement plus de temps (environ un quart d'heure) aux jeux et à l'instruction des enfants que les femmes non-enseignantes, ils en consacrent moins qu'elles aux activités de soins et d'accompagnement (cf. tableau 2a). Au total, le temps qu'ils consacrent aux enfants ne diffère donc pas de celui des femmes diplômées non enseignantes.

Ainsi, la profession d'enseignant entraîne un peu plus d'égalité quand on considère le temps total de la prise en charge des enfants, les jours de travail, les hommes enseignants consacrant plus de temps à leurs enfants que les autres hommes diplômés et un temps total comparable à celui des femmes diplômées non enseignantes. Néanmoins, nos résultats confirment ceux de Algava (2002) : les activités de «care » sont dévolues très majoritairement aux femmes, y compris quand les hommes sont enseignants. La plus grande prise en charge des enfants constatée pour les hommes enseignants un jour de travail étant limitée aux jeux et à l'instruction des enfants, la profession d'enseignant n'apparaît pas remettre en cause l'inégale répartition sexuée des tâches liées aux enfants. Et ceci d'autant plus que les jours de repos, la différence entre les hommes et les femmes s'accentue chez les enseignants pour qui, contrairement aux autres hommes, une augmentation du temps consacré aux enfants n'est pas constatée.

\section{Globalement, les enseignants consacrent plus de temps aux enfants, un jour de repos ou non}

Les différences liées aux normes de genre se maintiennent donc : les femmes en font plus, et c'est d'autant plus vrai pour les enseignantes.

Par ailleurs, les différences entre les enseignants et les non enseignants ne semblent pas être remises en cause par ce qui est observé les jours de repos. Une augmentation significative du temps passé à s'occuper des enfants les jours non travaillés est constatée seulement pour les diplômés non enseignants des deux sexes, ce qui serait cohérent avec l'hypothèse d'une légère compensation. Toutefois, dans la mesure où aucune différence ne peut être mise en évidence entre le temps consacré par les enseignants et les non enseignants des deux sexes, quel que soit le type de temps considéré, on ne peut pas véritablement conclure à un rééquilibrage mais plutôt à un léger rattrapage des inégalités observées. On peut donc considérer que les enseignants consacrent un peu plus de temps à leurs enfants que les autres diplômés, en prenant en compte tous les types de journées. La différence entre les diplômés non enseignants et les enseignants est d'autant plus accentuée que sur une année, les jours travaillés sont plus nombreux que les jours non travaillés.

\section{Les diplômés non enseignants ne compensent pas avec les activités secondaires}

Le différentiel de temps consacré aux enfants les jours de repos n'est donc pas suffisant pour conclure à une véritable compensation. La prise en compte des activités secondaires modifie-t-elle ce constat?

Les temps consacrés aux enfants déclarés en activités secondaires ont été ajoutés aux temps déclarés en activité principale (cf. tableau B en 
annexe). Les temps moyens se trouvent donc augmentés par rapport aux temps présentés dans le tableau $1^{14}$, sans toutefois remettre en question les variations observées entre catégories.

Par ailleurs, le fait de déclarer du temps pour les enfants en activité secondaire est ici toujours plus fréquent chez les femmes, qu'elles soient enseignantes ou non. Pour les enseignantes, on retrouve les résultats de Jarty (2009) qui décrit chez elles « un enchevêtrement des activités professionnelles et familiales » (p. 11), beaucoup plus rarement observé pour les hommes enseignants. Quand il existe de jeunes enfants dans la famille, ce phénomène est encore plus prononcé chez les enseignantes.

Les mêmes analyses que précédemment ont été réalisées sur ces totaux (cf. tableaux $\mathrm{C}$ et $\mathrm{D}$ en annexe), pour l'ensemble du temps ainsi que sur les temps consacrés aux jeux et à l'instruction des enfants d'une part, et aux soins et à l'accompagnement d'autre part.

Sur le temps total, ajouter les activités secondaires ne modifie pas les résultats obtenus sur le temps déclaré en activité principale. Quelques différences sont constatées pour le temps voué aux jeux et à l'instruction des enfants : quand les activités secondaires sont prises en compte, il s'avère que les femmes diplômées non enseignantes passent un peu plus de temps à ces activités que les hommes diplômés non enseignants, que le jour soit travaillé ou non, ce qui n'était pas observé sur les activités principales. L'autre différence concerne les hommes enseignants comparés aux femmes non enseignantes un jour de travail : alors que nous avons vu que les hommes enseignants consacrent plus de temps à ces activités que ces femmes un jour travaillé, la différence n'est plus significative quand les activités secondaires sont ajoutées ${ }^{15}$.

Pour les activités de soins et d'accompagnement, les variations observées sont du même type que celles constatées sur le temps déclaré en activité principale, mais les femmes déclarant plus de temps en activités secondaires que les hommes, les différences entre les hommes et les femmes sont plus accentuées.

Ces résultats ne permettent donc pas de considérer que le temps déclaré en activités secondaires compense les différences entre enseignants et diplômés non enseignants, ni les différences entre les hommes et les femmes, enseignants ou non. Ces dernières auraient même tendance à être majorées.

\section{Les enseignants en couple : le temps consacré aux enfants dépend essentiellement du sexe du parent et des compétences supposées}

Nous avons vu que le temps consacré aux jeux et à l'instruction des enfants est légèrement diminué quand le(la) conjoint(e) est enseignant(e), y compris quand les activités secondaires sont ajoutées (cf. tableaux $\mathrm{C}$ et $\mathrm{D}$ en annexe).

S'intéresser aux enseignants en couple permet maintenant de saisir si la profession d'enseignant est associée à une « spécialisation » dans l'éducation des enfants. Leurs activités sont étudiées en tenant compte à la fois du diplôme de leur conjoint(e) et de sa profession (enseignant ou non $)^{16}$.

Quand on compare les femmes enseignantes en couple avec un enseignant, à celles dont le conjoint n'est pas enseignant, on constate que le temps total consacré aux enfants par ces dernières est plus élevé (d'environ 20 minutes) seulement quand leur conjoint n'est pas diplômé de l'enseignement supérieur (cf. tableau E en annexe).

Cependant, 1'examen séparé du temps consacré aux jeux et à l'instruction, d'une part, et aux activités de soins et d'accompagnement, d'autre part, montre des résultats différents selon le niveau de diplôme du conjoint quand celui-ci n'est pas enseignant. Les femmes enseignantes dont le conjoint n'a pas fait d'études supérieures passent plus de temps aux activités de soins et d'accompagnement que les femmes enseignantes en couple avec un enseignant, mais pas plus de temps aux jeux et à l'éducation des enfants que ces dernières, alors que l'on aurait pu s'attendre à ce qu'elles prennent davantage en charge ces tâches quand leur conjoint est moins diplômé qu'elles. Une des explications pourrait être le caractère disparate du temps « consacré aux jeux et à l'instruction », composé à la fois d'activités ludiques et éducatives, ou une moindre importance accordée aux activités éducatives par ces couples que par les couples formés de deux conjoints fortement diplômés.

\footnotetext{
14. Les pourcentages de personnes ayant pratiqué l'activité sont un peu augmentés, certaines ayant déclaré du temps (même très peu) uniquement en activités secondaires. C'est pourquoi, sur ces personnes, des moyennes sont inférieures à celles du tableau 1.

15. La tendance va néanmoins dans le même sens et la probabilité est à la limite de la significativité statistique.

16. Le statut d'activité du conjoint n'est pas pris en compte, mais une forte majorité d'enseignant(e)s ont un(e) conjoint(e) en activité.
} 
Dans la mesure où les autres tâches domestiques qu'elles effectuent durent moins longtemps que pour les enseignantes dont le conjoint est enseignant (cf. tableau E en annexe), il est également possible que leur conjoint prenne en charge plus de tâches ménagères, et qu'elles se spécialisent davantage dans les soins aux enfants dans ces ménages. Ponthieux et Schreiber (2006) ont en effet montré que dans les couples de salariés, le taux de participation des hommes au noyau dur ${ }^{17}$ du travail domestique est un peu augmenté quand la femme gagne plus que son conjoint ${ }^{18}$.

Quant aux femmes enseignantes dont le conjoint est diplômé de l'enseignement supérieur sans être enseignant, elles vouent plus de temps aux jeux et à l'instruction des enfants que celles dont le conjoint est enseignant. Ces mères enseignantes dont le conjoint est souvent cadre, chef d'entreprise ou membre des professions libérales (dans $63 \%$ des cas) semblent se spécialiser dans l'éducation des enfants. Elles sont plus souvent à temps partiel que les autres enseignantes : $27 \%$ d'entre elles le sont, contre $22 \%$ des enseignantes dont le conjoint n'a pas de diplôme de l'enseignement supérieur, et $25 \%$ des enseignantes dont le conjoint est aussi enseignant. Elles passent moins de temps aux travaux ménagers que les enseignantes dont le conjoint est enseignant. Elles appartiennent à des ménages qui délèguent plus souvent les tâches ménagères à une aide rémunérée que les autres : ceci concerne $28 \%$ d'entre elles contre $19 \%$ des enseignantes dont le conjoint est aussi enseignant et seulement $10 \%$ des enseignantes dont le conjoint n'a pas de diplôme de l'enseignement supérieur.

Ces résultats semblent témoigner d'une prise en charge plus équilibrée des tâches éducatives réalisées par les couples diplômés quand les deux conjoints sont enseignants.

Quand on compare les hommes enseignants en couple avec une enseignante, à ceux dont la conjointe n'est pas enseignante, on constate que le temps total consacré aux enfants par ces derniers est plus élevé seulement quand leur conjointe n'est pas diplômée de l'enseignement supérieur (cf. tableau $\mathrm{F}$ en annexe). On retrouve le même résultat que chez les femmes. Néanmoins, cette différence est cette fois due au temps consacré aux jeux et à l'instruction, qui est augmenté d'environ 15 minutes. Gouyon et Guérin (2006), dans leur étude sur l'implication des parents dans la scolarité des filles et des garçons, avaient constaté que le suivi des devoirs obéissait «à une logique plutôt pragmatique, fonction des compétences et des disponibilités de chacun » (p. 70). La logique de la compétence associée à l'assignation de genre, pour ce type d'activités, semble plus forte que celle de la disponibilité car, dès lors que leur conjointe diplômée peut être considérée comme aussi compétente qu'eux, sans être aussi disponible, ils ne consacrent pas davantage de temps aux jeux et à l'instruction des enfants que ceux dont la femme est enseignante. En outre, les hommes enseignants consacrent moins de temps aux activités éducatives les jours où ils ne travaillent pas, ce qui laisse penser que lorsque les deux parents ont davantage de temps (si l'on suppose qu'il s'agisse également d'un jour de repos pour leur conjointe), ils la laissent prendre en charge ces activités.

Le temps consacré aux soins et à l'accompagnement des enfants, tout comme le temps consacré au « noyau dur » du travail domestique par les hommes enseignants en couple avec une femme non enseignante, n'est d'ailleurs pas différent de celui des enseignants en couple avec une enseignante. Ces résultats vont dans le même sens que ceux que montraient déjà Poissenot et de Singly (1993) dans leur étude sur les couples d'enseignants.

Par ailleurs, chez les hommes enseignants, l'exemple familial a une influence : ceux dont le père était enseignant consacrent plus de temps aux jeux et à l'instruction des enfants, et moins de temps aux soins des enfants que les autres. Comme mentionné supra dans le cas de la mère enseignante, il semble bien que l'on puisse mesurer là l'influence de modèles éducatifs transmis.

Ainsi, les logiques de genre, compétence et disponibilité se combinant au sein des couples, les femmes enseignantes consacrent plus de temps aux enfants que les autres individus.

\section{Les enseignants sont plus souvent et plus longtemps présents à leur domicile}

Dans quelle mesure cette disponibilité des enseignants est-elle effective, notamment à des moments où les enfants sont supposés être

17. Qui comprend la cuisine, le ménage, et le rangement et l'entretien du linge.

18. Dans $13 \%$ des cas, leur conjoint est cadre, chef d'entreprise ou membre des professions libérales, alors que ceci concerne $63 \%$ des enseignantes dont le conjoint est non enseignant, mais diplômé de l'enseignement supérieur. 
également présents - soit le soir et le mercredi ${ }^{19}$, cette « synchronisation » des temps familiaux pouvant être associée à des bénéfices scolaires (Henri-Panabière, 2010) ? S'intéresser à des temps et âges spécifiques se justifient également par les résultats de Jarty (2009), qui a mis en évidence que le «modèle de la féminitude » était le profil le plus fréquemment trouvé chez les femmes enseignantes, surtout avec des enfants petits et dans le cas de dissymétrie sociale du ménage. Selon l'auteure, ces femmes vantent le caractère flexible de leur profession, leur permettant de concilier facilement vie familiale et vie professionnelle, et « essayent autant que possible d'obtenir un emploi du temps calqué sur celui des enfants, l'obtention du "fameux » mercredi matin demeurant l'une des caractéristiques principales de la fiche de voux des mères de jeunes enfants appartenant à ce profil » (p. 12).

\section{La prise en charge des enfants scolarisés le soir et le mercredi}

La possibilité de bénéficier d'une présence parentale est d'autant plus importante qu'il y a des enseignants dans le ménage (cf. tableau 3 ). En effet, dans 8 cas sur 10 , dès qu'il y a un enseignant dans le couple, au moins un parent s'occupe du ou des enfants le soir et le mercredi. Les proportions augmentent encore et atteignent même $92 \%$ pour le soir et $93 \%$ pour le mercredi quand les deux conjoints sont enseignants. Dans les couples sans enseignant, cette proportion est de l'ordre de $70 \%$ des cas le soir

19. Un indicateur a été créé pour les différentes situations envisagées selon l'âge des enfants et le moment étudié, à partir des questions posées pour chaque enfant scolarisé à temps plein : " en général, qui s'occupe de "PRÉNOM » le soir / le mercredi après l'école ? (en considérant, d'une part, les enfants scolarisés de moins de 7 ans et, d'autre part, les enfants scolarisés de 7 à 14 ans) ». Les modalités de réponses étaient les suivantes : 1- La mère ; 2 - Le père ; les modalités 3 à 10 envisageaient les autres cas. Un parent s'occupant "en général » d'au moins un enfant le soir après l'école ou le mercredi a alors été considéré comme s'occupant des enfants à ces moments étudiés. Pour ce paragraphe, seuls seront considérés les ménages dans lesquels les deux membres du couple sont les parents des enfants concernés par les questions.

Tableau 3

Prise en charge des enfants scolarisés le soir et le mercredi, selon le nombre d'enseignant(s)

\begin{tabular}{|c|c|c|c|c|}
\hline & $\begin{array}{l}\text { Aucun enseignant } \\
\text { dans le ménage }\end{array}$ & $\begin{array}{l}\text { Seule la mère } \\
\text { est enseignante }\end{array}$ & $\begin{array}{l}\text { Seul le père } \\
\text { est enseignant }\end{array}$ & Deux enseignants \\
\hline \multicolumn{5}{|c|}{ Le soir après l'école } \\
\hline Aucun parent ${ }^{*}$ & 32 & 19 & 23 & 8 \\
\hline Un parent s'occupe des enfants & 67 & 79 & 73 & 89 \\
\hline En général, la mère s'occupe des enfants & 52 & 66 & 43 & 64 \\
\hline En général, le père s'occupe des enfants & 15 & 13 & 30 & 25 \\
\hline Les deux parents s'occupent des enfants & 1 & 2 & 4 & 3 \\
\hline \multicolumn{5}{|c|}{ Le mercredi } \\
\hline Aucun parent & 28 & 13 & 19 & 7 \\
\hline Un parent s'occupe des enfants & 72 & 85 & 80 & 86 \\
\hline En général, la mère s'occupe des enfants & 60 & 79 & 50 & 73 \\
\hline En général, le père s'occupe des enfants & 12 & 6 & 30 & 13 \\
\hline Les deux parents s'occupent des enfants & 0 & 2 & 1 & 7 \\
\hline
\end{tabular}

Note : * en fait, compte tenu de la manière dont est posée la question, une seule personne étant proposée, la modalité « les deux parents " signifie que chaque parent s'occupe d'un enfant différent, et la modalité « aucun parent » correspond à une autre personne de la liste proposée (grands-parents, d'autres personnes de la famille, amis, voisins, solution de garde collective, assistante maternelle, nourrice ou baby-sitter, ainsi que pour les plus grands, "il reste seul ou avec des copains »). À ces modalités évoquant ces diverses solutions s'ajoute une modalité "Autres ». II n'est pas exclu que certains parents gardant ensemble leur(s) enfant(s) aient choisi cette modalité. Cependant même si c'est le cas, cela modifierait peu les résultats car ceci concerne, pour le soir, $2 \%$ des réponses quand il n'y a aucun enseignant, $1 \%$ quand seule la mère est enseignante, $3 \%$ quand seul le père est enseignant, et $1 \%$ quand les deux parents sont enseignants ; et, pour le mercredi, $0 \%$ des réponses quand il n'y a aucun enseignant, $2 \%$ quand seule la mère est enseignante, $4 \%$ quand seul le père est enseignant, et $1 \%$ quand les deux parents sont enseignants.

Lecture : quand aucun des parents n'est enseignant, dans $52 \%$ des cas, c'est la mère qui s'occupe du ou des enfant(s) le soir. C'est vrai dans $64 \%$ des cas quand les deux parents sont enseignants.

Champ : enseignants et diplômés de l'enseignement supérieur (titulaires d'au moins une licence), ayant une activité professionnelle et vivant en couple dans un ménage dans lequel il existe au moins un enfant scolarisé de moins de 15 ans, tous les enfants de moins de 15 ans du ménage étant les enfants des deux membres du couple.

Source : enquête Emploi du temps 2010-2011. 
comme le mercredi ${ }^{20}$; ce résultat est voisin de celui obtenu par Brown (2007) à partir de l'enquête Éducation et famille.

Par ailleurs, tenir compte du nombre d'enseignant(s) dans le couple permet d'étudier si le fait que les deux parents soient enseignants introduit plus d'égalité dans la prise en charge des enfants. Très majoritairement, c'est seulement la mère qui prend en charge les enfants le soir (64 \%) et le mercredi (73\%), même quand les deux parents sont enseignants. Néanmoins, la prise en charge des enfants par uniquement le père devient un peu plus importante le soir (25\% contre $13 \%$ le mercredi).

Enfin, si l'on spécifie par rapport à l'âge des enfants, on constate que cette spécialisation genrée des rôles est d'autant plus importante que les enfants scolarisés sont jeunes (moins de 7 ans). Dans ce cas, elle est particulièrement accentuée le mercredi dans les couples d'enseignants : dans $82 \%$ des cas, c'est la mère seulement qui s'occupe des enfants, et dans $10 \%$ des cas, le père. Le soir, la mère s'occupe généralement des jeunes enfants dans $71 \%$ des cas, puis le père dans $21 \%$ des cas.

À l'inverse, quand les deux parents sont enseignants, et quand les enfants sont plus âgés, la prise en charge des enfants devient un peu plus égalitaire, en particulier le soir. Les enfants de 7 à 14 ans sont pris en charge par leur mère dans $74 \%$ des cas, le père dans $14 \%$ des cas et les deux dans $7 \%$ des cas (avec dans $5 \%$ des cas, aucun des deux) le mercredi. Pour des enfants de 7 à 14 ans, le soir, dans $55 \%$ des cas, c'est la mère, dans $31 \%$, le père, et pour $1 \%$, les deux parents.

\section{Les enseignants, davantage présents à leur domicile}

Si l'on se penche sur la disponibilité des parents, mesurée cette fois à travers leur temps de présence au domicile (cf. tableau 4), les enseignants sont présents environ 2 heures de plus à leur domicile, pour une journée quelconque (travaillée ou non), que les non-enseignants. La différence est plus accentuée chez les hommes (environ $2 \mathrm{~h} 30$ de plus) que chez les femmes (environ 1h30). En se restreignant à la disponibilité après $17 \mathrm{~h}$, on constate que là encore, les enseignants passent plus de temps à leur domicile que les non-enseignants (environ trois quarts d'heure), avec une différence de presque 1 heure chez les hommes. Ainsi, les enseignants sont bien plus présents à leur domicile, après la fin du temps scolaire, ce qui favorise un accompagnement des activités des enfants, et notamment des activités scolaires.

En s'intéressant plus spécifiquement aux heures qui correspondent à la fin du temps scolaire (à partir de $17 \mathrm{~h}$, cf. figure $)^{21}$, on observe une plus grande disponibilité des enseignants dès $17 \mathrm{~h}$ (plage horaire : 17h-20h), qui persiste au cours de la soirée (plages horaires : 18h-20h et 19h-20h). Si un quart d'entre eux est présent au domicile sur la plage horaire $17 \mathrm{~h}-20 \mathrm{~h}$, ceci concerne seulement $15 \%$ des non-enseignants ; $70 \%$ des premiers sont chez eux à partir de $19 \mathrm{~h}$, quand c'est le cas d'à peine plus de la moitié des seconds.

Par ailleurs, la différence entre les hommes et les femmes est ténue mais s'accentue de $19 \mathrm{~h}$ à $20 \mathrm{~h}$, chez les enseignants comme les non-enseignants.

20. La proportion élevée de femmes diplômées non-enseignantes s'occupant généralement d'un enfant le mercredi inviterait à prendre en compte le temps partiel. Malheureusement à la suite d'un problème de recueil pour cette information, cela n'a pas été possible car elle comporte un grand nombre de données manquantes, pour les non-enseignants. Pour ces derniers, l'information n'est ainsi pas disponible pour $19 \%$ des femmes et $17 \%$ des hommes.

21. Ce taux de présence est éventuellement un peu sous-estime car est considéré comme au domicile, sur la plage horaire étudiée, une personne présente de manière continue.

\section{Tableau 4}

Temps de présence au domicile, toutes journées confondues

\begin{tabular}{|l|c|c|c|c|c|c|}
\cline { 2 - 7 } \multicolumn{1}{c|}{} & \multicolumn{3}{c|}{ Enseignants } & \multicolumn{3}{c|}{ Diplômés non enseignants } \\
\cline { 2 - 7 } \multicolumn{1}{c|}{} & Hommes & Femmes & Ensemble & Hommes & Femmes & Ensemble \\
\hline Sur l'ensemble de la journée & $16 \mathrm{~h} 47$ & $16 \mathrm{~h} 54$ & $16 \mathrm{~h} 52$ & $14 \mathrm{~h} 19$ & $15 \mathrm{~h} 29$ & $14 \mathrm{~h} 52$ \\
Le soir après 17h & $5 \mathrm{~h} 10$ & $5 \mathrm{~h} 21$ & $5 \mathrm{~h} 19$ & $4 \mathrm{~h} 19$ & $4 \mathrm{~h} 49$ & $4 \mathrm{~h} 33$ \\
\hline
\end{tabular}

Lecture : les hommes enseignants sont présents en moyenne $16 \mathrm{~h} 47$ à leur domicile sur l'ensemble de la journée, contre $14 \mathrm{~h} 19$ pour les hommes non enseignants.

Champ : enseignants et diplômés de l'enseignement supérieur (titulaires d'au moins une licence), ayant une activité professionnelle et vivant dans un ménage dans lequel il existe au moins un enfant de moins de 18 ans.

Source : enquête Emploi du temps 2010-2011. 
On peut avancer que la présence au domicile ne signifie pas automatiquement la prise en charge des enfants ; néanmoins, le fait d'être présents plus tôt au domicile donne la possibilité aux enseignants d'exercer un plus grand contrôle parental, ce qui va dans le sens des constats de Henri-Panabière (2010), et Millet et Thin (2005).

\section{Parmi les sur-investis, plus d'enseignants...}

Nous avons constaté que les enseignants consacrent un peu plus de temps aux enfants que les autres diplômés. Nous nous sommes alors demandé si cet effet « parent enseignant » se maintenait en s'intéressant aux individus qui passent le plus de temps dans des activités à destination des enfants, que ce soit des jeux, des loisirs, des soins, etc. Autrement dit, les effets observés en moyenne se maintiennent-ils quand on s'intéresse aux bornes supérieures : les enseignants ont-ils aussi plus de chances de faire partie de ceux qui se " surinvestissent " auprès de leurs enfants ${ }^{22}$ ?

Quand les enfants sont jeunes, $61 \%$ des enseignants et $45 \%$ des diplômés non enseignants consacrent plus de 1 h30 par jour aux enfants. Ces proportions passent respectivement à $26 \%$ et $19 \%$ quand les enfants ont plus de six ans. Les différences entre enseignants et non-enseignants sont particulièrement prononcées pour les femmes quand les enfants sont jeunes. En effet, $67 \%$ des enseignantes consacrent beaucoup de temps aux enfants, contre $53 \%$ des non-enseignantes.

Les comparaisons entre les différentes situations toutes choses égales par ailleurs ont été encore réalisées en tenant compte simultanément du sexe, du fait d'être enseignant ou non, et du jour travaillé ou non, en faisant varier la situation de référence du modèle. Les résultats sont donnés dans le tableau $\mathrm{G}$ (en annexe) en prenant comme situation de référence « femme non enseignante un jour travaillé » et dans le tableau $\mathrm{H}$ (en annexe) en prenant comme situation de référence « homme non enseignant un jour travaillé ». Cette analyse confirme que les enseignants des deux sexes ont plus de chances de faire partie des personnes qui consacrent le plus de temps à leurs enfants que les hommes ou les femmes diplômés non enseignants un jour travaillé. Ce n'est plus le cas lorsqu'ils

22. La probabilité de faire partie de ceux qui consacrent le plus de temps total aux enfants a été modélisée. Sont dans ce groupe les individus appartenant au tercile supérieur pour le temps consacré aux enfants (c'est-à-dire plus de 1h30). Pour étudier l'effet de la profession (enseignant ou non) sur cette probabilité, en tenant compte des autres caractéristiques considérées comme paramètres explicatifs $V_{p}$ nous avons eu recours à une régression logistique. Faire partie de ce groupe a été considéré de manière dichotomique (prenant la valeur 1 si c'est le cas, et 0 dans le cas contraire).

\section{Figure \\ Taux de présence au domicile de $17 \mathrm{~h}$ à $20 \mathrm{~h}$}

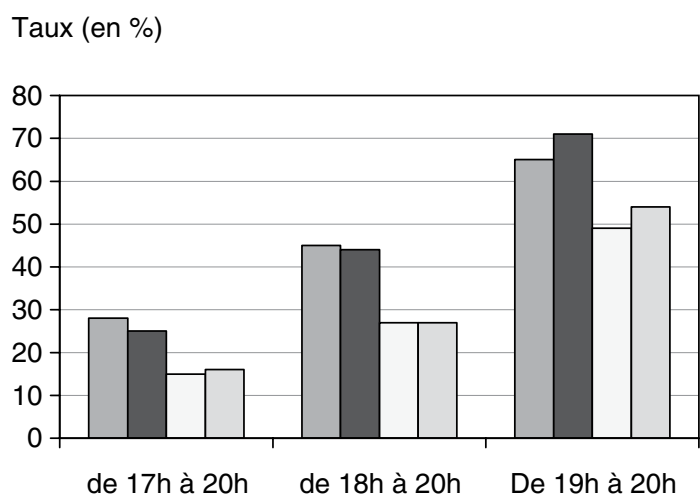

\begin{tabular}{|l}
$\square$ Hommes enseignants $\quad \square$ Femmes enseignantes \\
$\square$ Hommes non enseignants $\square$ Femmes non enseignantes
\end{tabular}

Lecture : $28 \%$ des hommes enseignants sont présents à leur domicile de $17 \mathrm{~h}$ à $20 \mathrm{~h}$; c'est le cas de $25 \%$ des femmes enseignantes. Champ : enseignants et diplômés de l'enseignement supérieur (titulaires d'au moins une licence), ayant une activité professionnelle et vivant dans un ménage dans lequel il existe au moins un enfant de moins de 18 ans.

Source : enquête Emploi du temps 2010-2011. 
ne travaillent pas : ils n'ont alors pas plus de chances que les non-enseignants de faire partie de ce groupe. Une spécificité est toutefois constatée chez les hommes : les chances de faire partie de ceux qui consacrent le plus de temps aux enfants augmentent alors pour les nonenseignants, tandis que l'inverse est constaté pour les hommes enseignants. Ainsi, les hommes enseignants s'investiraient particulièrement dans leurs activités parentales les jours travaillés, et un peu moins les jours de repos.

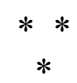

L'hypothèse selon laquelle l'organisation temporelle spécifique des enseignants est plus favorable à la réussite scolaire des enfants n'est pas rejetée par cette étude. En effet, les enseignants sont plus souvent et plus longtemps présents à leur domicile que les autres diplômés du supérieur, et consacrent globalement plus de temps à leurs enfants que ces derniers. Mais derrière ce constat subsistent des différences importantes entre les sexes, et selon le type de temps et de journée étudié. Il semblerait qu'il existe plutôt un " effet mère enseignante ", qu'un " effet parent enseignant $»$.

$\mathrm{Si}$ on compare les hommes entre eux, et les femmes entre elles, le fait d'être enseignant entraîne une augmentation du temps consacré aux enfants les jours travaillés. Pour les femmes, ceci est vrai quel que soit le type de temps étudié. Pour les hommes, ceci est constaté uniquement pour le temps voué aux jeux et à l'instruction. Ces différences entre enseignants et autres diplômés du supérieur ne sont pas compensées les jours de repos, car les hommes et les femmes diplômés non enseignants, s'ils augmentent alors leur temps parental, ne s'investissent pas davantage dans des activités avec les enfants que les hommes et les femmes enseignants.

Si on compare les enseignants entre eux, et les non-enseignants entre eux, on constate que les différences entre les sexes persistent : quelle que soit la journée considérée, les activités liées aux soins et à l'accompagnement des enfants sont majoritairement dévolues aux femmes, enseignantes ou non. La profession enseignante ne paraît donc pas aboutir à une distribution moins genrée des tâches.

Alors même que les jours travaillés, les hommes enseignants sont présents à leur domicile plus tôt que les femmes diplômées non enseignantes, cette plus grande disponibilité ne se traduit pas par une prise en charge égalitaire des enfants. Les hommes enseignants consacrent en effet un peu plus de temps que les femmes nonenseignantes aux jeux et à l'instruction des enfants, mais toujours moins de temps aux soins et à l'accompagnement.

\section{BIBLIOGRAPHIE}

Algava E. (2002), « Quel temps pour les activités parentales? », Études et Résultats, $\mathrm{n}^{\circ} 162$, mars.

Anxo D., Flood L. et Kocoglu Y. (2002), « Offre de travail et répartition des activités domestiques et parentales au sein du couple : une comparaison entre la France et la Suède », Économie et statistique, $\mathrm{n}^{\circ} 352-353$, pp. 127-150.

Battaglia M. (2013), « Enfants d'enseignants, ces «chouchous» de l'école», Le Monde, 08 mai 2013.

Baudelot C. et Establet R. (2009), L'élitisme républicain. L'école française à l'épreuve des comparaisons internationales, Éditions du Seuil et La République des Idées, Paris.
Bouhia R. (2005), « Les étudiants en classes préparatoires aux grandes écoles. Année 2004$2005 »$, Note d'Information 05.22, MEN-DEP, septembre.

Bourdieu P. et Passeron J.-C. (1964), Les héritiers. Les étudiants et la culture, Coll. Le sens commun, Les Éditions de Minuit, Paris.

Bourdieu P. (1979), « Les trois états du capital culturel », Actes de la recherche en sciences sociales, vol. 30, novembre, pp. 3-6.

Brown É. (2007), "Les contributions des pères et des mères à l'éducation des enfants", Revue française des affaires sociales, vol. $1, \mathrm{n}^{\circ} 1$, pp. 127-151. 
Brugeilles C. et Sebille P. (2009), « La participation des pères aux soins et à l'éducation des enfants. L'influence des rapports sociaux de sexe entre les parents et entre les générations », Politiques sociales et familiales, $\mathrm{n}^{\circ}$ 95, mars, pp. 19-32.

Caro P. et Hillau B. (1997), « La logique dominante des publics scolaires. Offre de formation et environnement local », Formation Emploi, $\mathrm{n}^{\circ}$ 59, juillet-septembre, pp. 87-103.

Chenu A. (2002), « Les horaires et l'organisation du temps de travail », Économie et Statistique, n³52-353, pp. 151-167.

Da-Costa Lasne A. (2012), La singulière réussite scolaire des enfants d'enseignants : des pratiques éducatives parentales spécifiques? Thèse de doctorat en sciences de l'éducation, sous la direction de Marie Duru-Bellat, Université de Bourgogne, Dijon.

DEP (2003), « L'école réduit-elle les inégalités sociales ? », Éducation \& formations, $\mathrm{n}^{\circ} 66$, juillet-décembre, pp. 177-185.

Farges G. (2010), Les identités enseignantes à l'épreuve du temps. Les transformations intergénérationnelles d'un groupe social (1970-2010). Thèse de doctorat en sociologie, sous la direction de Louis Chauvel, Institut d'études politiques de Paris, Paris.

Farges G. (2011), « Le statut social des enseignants français au prisme du renouvellement générationnel ", Revue européenne des sciences sociales, $\mathrm{n}^{\circ} 49.1$, pp. 157-178.

Gouyon M. et Guérin S. (2006), « L'implication des parents dans la scolarité des filles et des garçons : des intentions à la pratique », Économie et Statistique, n 398-399, pp. 59-84.

Henri-Panabière G. (2010), Des " héritiers » en échec scolaire, Paris, La Dispute.

Henriot-Van Zanten A. (1996), « Stratégies utilitaristes et stratégies identitaires des parents vis-à-vis de l'école : une relecture critique des analyses sociologiques "), Lien social et Politiques, $\mathrm{n}^{\circ} 35$, printemps, pp. 125-135.
Héran F. (1996), « École publique, école privée : qui peut choisir? », Économie et Statistique, $\mathrm{n}^{\circ} 293$, pp. 17-39.

Jarty J. (2009), « Les usages de la flexibilité temporelle chez les enseignants du secondaire », Temporalités, $\mathrm{n}^{\circ} 9$, mis en ligne le 30 septembre 2009 (consulté le 29 mai 2013). URL : http://temporalites.revues.org/1057

Millet M. et Thin D. (2005), Ruptures scolaires. L'école à l'épreuve de la question sociale, Puf, Paris.

MENERS (2011), Repères et références statistiques sur les enseignements, la formation et la recherche (RERS), MEN-DEPP, septembre.

Poissenot Cl. et Singly de F. (1993), « Les enseignants en couple ", Éducation \& formations, $\mathrm{n}^{\circ} 46$, juillet, pp. 93-105.

Ponthieux S. et Schreiber A. (2006), « Dans les couples de salariés, la répartition du travail domestique reste inégale », Données sociales, Insee, Paris.

Ricroch L. et Roumier B. (2011), " Depuis 11 ans, moins de tâches ménagères, plus d'Internet », Insee Première, $n^{\circ} 1377$, novembre.

Sautory O. (2007), « La démocratisation de l'enseignement supérieur : évolution comparée des caractéristiques sociodémographiques des bacheliers et des étudiants ", Éducation \& formations, $\mathrm{n}^{\circ} 74$, avril, pp. 49-64.

Thin D. (1998), Quartiers populaires. L'école et les familles, Pul, Lyon.

Vanhoeffelen A. (2010), « Les bacheliers du panel 1995 : évolution et analyse des parcours », Note d'information 10-13, MEN-DEPP, septembre.

Van Zanten A. (2009), « Le travail éducatif parental dans les classes moyennes et supérieures : deux modes contrastés d'encadrement des pratiques et des choix des enfants », Informations Sociales, $\mathrm{n}^{\circ} 154$, pp. $80-87$. 
Tableau A

Caractéristiques comparées des deux populations

En \%

\begin{tabular}{|c|c|c|c|}
\hline & Enseignants & $\begin{array}{c}\text { Diplômés } \\
\text { non enseignants }\end{array}$ & Ensemble \\
\hline \multicolumn{4}{|l|}{ Sexe } \\
\hline Hommes & 29,7 & 49,6 & 47,0 \\
\hline Femmes & 70,3 & 50,4 & 53,0 \\
\hline \multicolumn{4}{|l|}{ Classe d'âge } \\
\hline$<35$ ans & 18,7 & 26,4 & 25,3 \\
\hline 35 à 44 ans & 60,1 & 52,0 & 53,0 \\
\hline 45 ans et + & 21,3 & 21,7 & 21,6 \\
\hline \multicolumn{4}{|l|}{ Diplôme } \\
\hline Au plus $\mathrm{Bac}+2$ & 18,8 & & 2,5 \\
\hline Deuxième cycle & 62,4 & 40,1 & 43,1 \\
\hline Grande École & 2,2 & 21,3 & 18,7 \\
\hline Troisième cycle & 16,5 & 38,6 & 35,7 \\
\hline \multicolumn{4}{|l|}{ Type de ménage } \\
\hline Famille monoparentale & 8,2 & 8,8 & 8,7 \\
\hline En couple avec enfant(s) et le conjoint n'est pas enseignant & 60,9 & 81,9 & 79,1 \\
\hline En couple avec enfant(s) et le conjoint est enseignant & 30,9 & 9,3 & 12,2 \\
\hline \multicolumn{4}{|l|}{ Nombre et âge des enfants } \\
\hline Un seul enfant d'âge $\leq 6$ ans & 12,0 & 22,2 & 20,8 \\
\hline Deux enfants et + (au moins deux d'âge $\leq 6$ ans) & 9,8 & 9,8 & 9,8 \\
\hline Deux enfants et + (dont un d'âge $\leq 6$ ans) & 20,4 & 23,2 & 22,8 \\
\hline Deux enfants et + (d'âges $>6$ ans) & 33,3 & 24,1 & 25,3 \\
\hline Un seul enfant âgé de plus de 6 ans (et de moins de 18 ans) & 24,4 & 20,8 & 21,2 \\
\hline \multicolumn{4}{|l|}{ Diplôme du conjoint si en couple } \\
\hline$<\mathrm{Bac}$ & 16,4 & 13,8 & 14,2 \\
\hline Brevet professionnel à Bac & 12,1 & 11,7 & 11,7 \\
\hline $\mathrm{Bac}+2$ & 20,8 & 17,4 & 17,9 \\
\hline Deuxième cycle & 28,1 & 21,0 & 22,0 \\
\hline Troisième cycle ou grande école & 22,6 & 36,0 & 34,3 \\
\hline \multicolumn{4}{|l|}{ Milieu d'origine } \\
\hline Mère enseignante & 14,2 & 12,7 & 12,9 \\
\hline Père enseignant & 11,3 & 6,7 & 7,3 \\
\hline \multicolumn{4}{|l|}{ Diplôme du père } \\
\hline$<\mathrm{Bac}$ & 55,7 & 50,1 & 50,8 \\
\hline Bac et plus & 35,2 & 42,0 & 41,1 \\
\hline Non renseigné & 9,1 & 7,9 & 8,1 \\
\hline \multicolumn{4}{|l|}{ Diplôme de la mère } \\
\hline$<\mathrm{Bac}$ & 59,5 & 50,1 & 51,3 \\
\hline Bac et plus & 31,8 & 42,0 & 40,7 \\
\hline Non renseigné & 8,7 & 7,9 & 8,0 \\
\hline \multicolumn{4}{|l|}{ Type de commune } \\
\hline Rurale à moins de 20000 hab. & 43,8 & 23,9 & 26,6 \\
\hline 20000 hab. à moins de 200000 hab. & 22,5 & 17,8 & 18,4 \\
\hline 200000 hab. et + & 33,7 & 58,3 & 55,0 \\
\hline Effectifs & 552 & 766 & 1318 \\
\hline
\end{tabular}

Lecture : 14,2 \% des enseignants ont une mère elle-même enseignante. C'est le cas de 12,7 \% des diplômés non enseignants. Champ : enseignants et diplômés de l'enseignement supérieur (titulaires d'au moins une licence), ayant une activité professionnelle et vivant dans un ménage dans lequel il existe au moins un enfant de moins de 18 ans.

Source : enquête Emploi du temps 2010-2011. 


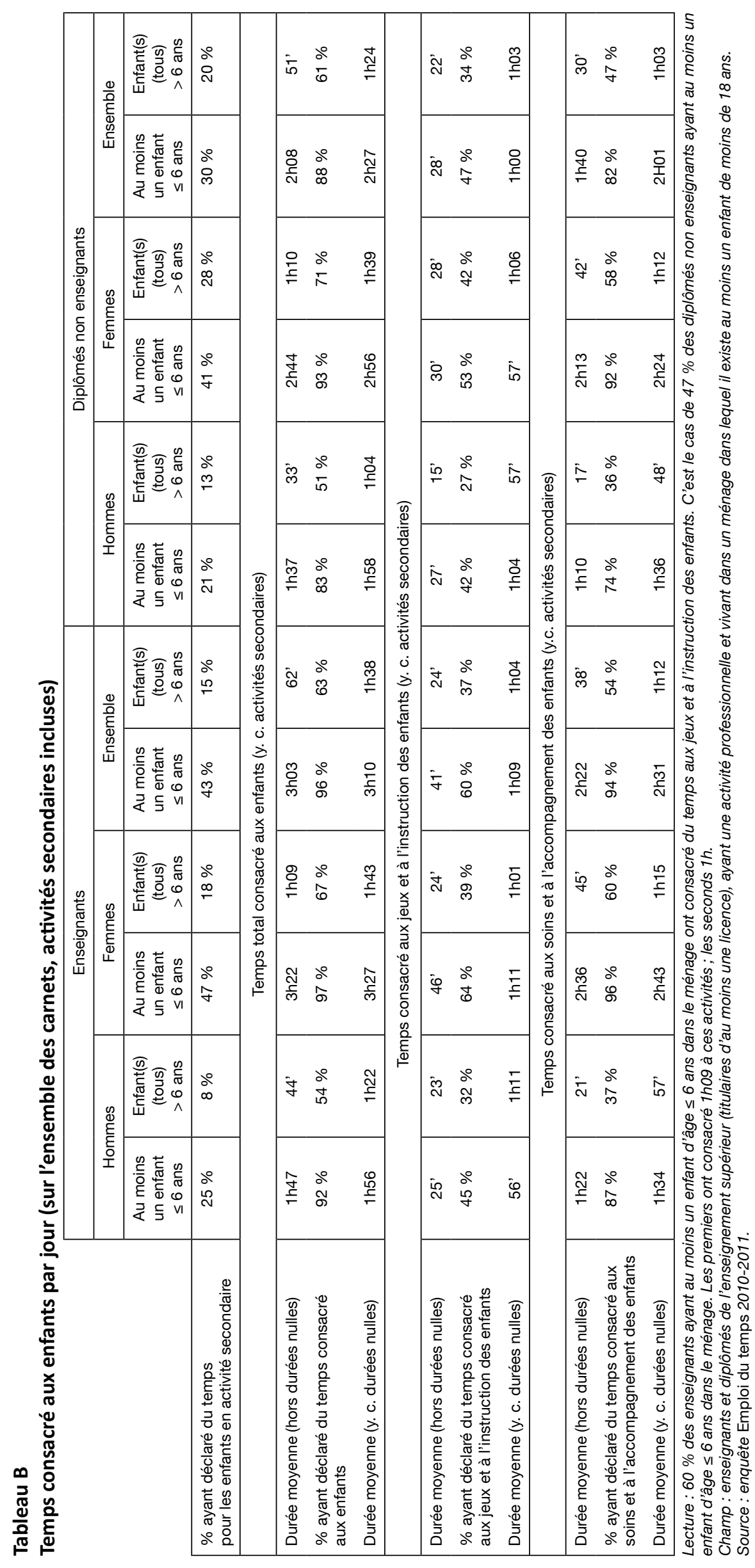


Tableau C

Temps consacré aux enfants (y. c. activités secondaires). Résultats des estimations des modèles Tobit

(Référence : femme diplômée non enseignante un jour travaillé)

\begin{tabular}{|c|c|c|c|c|c|c|}
\hline Coefficients, effets marginaux et valeurs de $p$ & \multicolumn{2}{|c|}{ Temps total } & \multicolumn{2}{|c|}{$\begin{array}{l}\text { Temps consacré } \\
\text { aux jeux } \\
\text { et à l'instruction }\end{array}$} & \multicolumn{2}{|c|}{$\begin{array}{l}\text { Temps consacré } \\
\text { aux soins et à } \\
\text { l'accompagnement }\end{array}$} \\
\hline \% concernés par l'activité & \multirow{2}{*}{\multicolumn{2}{|c|}{$\begin{array}{c}73 \% \\
96\end{array}$}} & \multirow{2}{*}{\multicolumn{2}{|c|}{$\begin{array}{c}35 \% \\
26 \\
\end{array}$}} & \multirow{2}{*}{\multicolumn{2}{|c|}{$\begin{array}{c}67 \% \\
70 \\
\end{array}$}} \\
\hline Moyennes sur l'ensemble & & & & & & \\
\hline & Coefficient & $\begin{array}{c}\text { Effet } \\
\text { marginal }\end{array}$ & Coefficient & $\begin{array}{c}\text { Effet } \\
\text { marginal }\end{array}$ & Coefficient & $\begin{array}{c}\text { Effet } \\
\text { marginal }\end{array}$ \\
\hline \multicolumn{7}{|l|}{ Sexe et profession et type de journée } \\
\hline Femme enseignante un jour travaillé & $+35^{\star \star \star}$ & +25 & $+24^{\star \star \star}$ & +10 & $+23^{\star \star}$ & +15 \\
\hline Femme enseignante un jour non travaillé & $+56^{\star \star \star}$ & +39 & $+34^{\star \star \star}$ & +15 & $+35^{\star \star \star}$ & +22 \\
\hline Femme diplômée non enseignante un jour travaillé & Réf. & & Réf. & & Réf. & \\
\hline $\begin{array}{l}\text { Femme diplômée non enseignante un jour } \\
\text { non travaillé }\end{array}$ & $+64^{\star \star \star}$ & +45 & $+33^{\star \star \star}$ & +14 & $+44^{\star \star \star}$ & +27 \\
\hline Homme enseignant un jour travaillé & -14 & & +24 & & $-44^{\star \star}$ & -27 \\
\hline Homme enseignant un jour non travaillé & $-47^{*}$ & -33 & -8 & & $-52^{\star \star}$ & -32 \\
\hline Homme diplômé non enseignant un jour travaillé & $-54^{\star \star \star}$ & -38 & $-17^{\star \star \star}$ & -7 & $-58^{\star \star \star}$ & -36 \\
\hline $\begin{array}{l}\text { Homme diplômé non enseignant un jour } \\
\text { non travaillé }\end{array}$ & -9 & & $+17^{\star \star}$ & +7 & $-30^{\star \star \star}$ & -19 \\
\hline \multicolumn{7}{|l|}{ Type de ménage } \\
\hline Famille monoparentale & +16 & & -5 & & $+23^{\star \star}$ & +14 \\
\hline $\begin{array}{l}\text { En couple avec enfant(s) et le conjoint } \\
\text { n'est pas enseignant }\end{array}$ & Réf. & & Réf. & & Réf. & \\
\hline $\begin{array}{l}\text { En couple avec enfant(s) et le conjoint } \\
\text { est enseignant }\end{array}$ & +1 & & $-15^{\star \star}$ & -6 & +11 & \\
\hline \multicolumn{7}{|l|}{ Nombre et âge des enfants } \\
\hline Un seul enfant d'âge $\leq 6$ ans & $+123^{\star \star \star}$ & +87 & $+15^{\star}$ & +6 & $+139^{\star \star \star}$ & +87 \\
\hline Deux enfants et plus (au moins deux d'âge $\leq 6$ ans) & $+203^{\star \star \star}$ & +143 & $+42^{\star \star \star}$ & +18 & $+208^{\star \star \star}$ & +130 \\
\hline Deux enfants et plus (dont un d'âge $\leq 6$ ans) & $+100^{\star \star \star}$ & +71 & -1 & & $+127^{\star \star \star}$ & +80 \\
\hline Deux enfants et plus d'âge $>6$ ans & $+43^{\star \star \star}$ & +30 & -1 & & $+61^{\star \star \star}$ & +38 \\
\hline $\begin{array}{l}\text { Un seul enfant âgé de plus de } 6 \text { ans (et de moins } \\
\text { de } 18 \text { ans) }\end{array}$ & Réf. & & Réf. & & & \\
\hline \multicolumn{7}{|l|}{ Classes d'âge } \\
\hline$<35$ ans & $+25^{\star \star}$ & +17 & $+25^{\star \star \star}$ & +11 & $+19^{*}$ & +12 \\
\hline 35 à 44 ans & $+39^{\star \star \star}$ & +27 & $+32^{\star \star \star}$ & +14 & $+28^{\star \star \star}$ & +18 \\
\hline 45 ans et + & Réf. & & Réf. & & Réf. & \\
\hline \multicolumn{7}{|l|}{ Parent(s) enseignant(s) } \\
\hline Mère enseignante & +11 & & +1 & & $+16^{\star \star}$ & +10 \\
\hline Mère non enseignante & Réf. & & Réf. & & Réf. & \\
\hline Père enseignant & -12 & & +4 & & -17 & \\
\hline Père non enseignant & Réf. & & Réf. & & Réf. & \\
\hline \multicolumn{7}{|l|}{ Type de commune } \\
\hline Rurale à moins de 20000 hab. & Réf. & & Réf. & & Réf. & \\
\hline 20000 hab. à moins de 200000 hab. & +10 & & $+19^{\star \star \star}$ & +8 & +3 & \\
\hline 200000 hab. et + & $+19^{\star \star \star}$ & +13 & $+30^{\star \star \star}$ & +13 & -2 & \\
\hline
\end{tabular}

Note : ${ }^{* \star}$ significatif à $1 \% ;{ }^{* *}$ à $5 \%$; ${ }^{*}$ à $10 \%$; - : non significatif ; Réf. : catégorie de référence. Les effets marginaux sont précisés seulement quand le coefficient est significatif.

Lecture : un jour travaillé, les femmes enseignantes consacrent 39 minutes de plus aux enfants que les femmes diplômées non enseignantes.

Champ : enseignants et diplômés de l'enseignement supérieur (titulaires d'au moins une licence), ayant une activité professionnelle et vivant dans un ménage dans lequel il existe au moins un enfant de moins de 18 ans.

Source : enquête Emploi du temps 2010-2011. 
Tableau D

Temps consacré aux enfants (y. c. activités secondaires). Résultats des estimations des modèles Tobit

(Référence : homme diplômé non enseignant un jour travaillé)

\begin{tabular}{|c|c|c|c|c|c|c|}
\hline Coefficients, effets marginaux et valeurs de $p$ & \multicolumn{2}{|c|}{ Temps total } & \multicolumn{2}{|c|}{$\begin{array}{l}\text { Temps consacré aux } \\
\text { jeux et à l'instruction }\end{array}$} & \multicolumn{2}{|c|}{$\begin{array}{c}\text { Temps consacré aux } \\
\text { soins et à l'accompa- } \\
\text { gnement }\end{array}$} \\
\hline \% concernés par l'activité & \multirow{2}{*}{\multicolumn{2}{|c|}{$\begin{array}{l}73 \% \\
96\end{array}$}} & \multirow{2}{*}{\multicolumn{2}{|c|}{$\begin{array}{c}35 \% \\
26 \\
\end{array}$}} & \multirow{2}{*}{\multicolumn{2}{|c|}{$\begin{array}{c}67 \% \\
70 \\
\end{array}$}} \\
\hline Moyennes sur l'ensemble & & & & & & \\
\hline & Coefficient & $\begin{array}{c}\text { Effet } \\
\text { marginal }\end{array}$ & Coefficient & $\begin{array}{l}\text { Effet } \\
\text { marginal }\end{array}$ & Coefficient & $\begin{array}{l}\text { Effet } \\
\text { marginal }\end{array}$ \\
\hline \multicolumn{7}{|l|}{ Sexe et profession et type de journée } \\
\hline Femme enseignante un jour travaillé & $+89^{\star \star \star}$ & +63 & $+41^{\star \star \star}$ & +18 & $+81^{\star \star *}$ & +51 \\
\hline Femme enseignante un jour non travaillé & $+110^{\star \star \star}$ & +78 & $+51^{\star \star \star}$ & +22 & $+93^{\star * \star}$ & +58 \\
\hline Femme diplômée non enseignante un jour travaillé & $+54^{\star \star \star}$ & +38 & $+17^{\star \star \star}$ & +7 & $+58^{\star \star \star}$ & +36 \\
\hline $\begin{array}{l}\text { Femme diplômée non enseignante un jour } \\
\text { non travaillé }\end{array}$ & $+118^{\star \star \star}$ & +84 & $+50^{\star \star \star}$ & +21 & $+102^{\star \star \star}$ & +64 \\
\hline Homme enseignant un jour travaillé & $+40^{\star \star}$ & +28 & $+41^{\star \star \star}$ & +18 & +14 & \\
\hline Homme enseignant un jour non travaillé & +7 & & +10 & & +7 & \\
\hline Homme diplômé non enseignant un jour travaillé & Réf. & & Réf. & & Réf. & \\
\hline $\begin{array}{l}\text { Homme diplômé non enseignant un jour } \\
\text { non travaillé }\end{array}$ & $+45^{\star \star \star}$ & +32 & $+34^{\star \star \star}$ & +15 & $+28^{\star \star \star}$ & +18 \\
\hline \multicolumn{7}{|l|}{ Type de ménage } \\
\hline Famille monoparentale & +16 & & -5 & & $+23^{\star \star}$ & +14 \\
\hline $\begin{array}{l}\text { En couple avec enfant(s) et le conjoint n'est pas } \\
\text { enseignant }\end{array}$ & Réf. & & Réf. & & Réf. & \\
\hline $\begin{array}{l}\text { En couple avec enfant(s) et le conjoint } \\
\text { est enseignant }\end{array}$ & +1 & & $-15^{\star \star}$ & -6 & +11 & \\
\hline \multicolumn{7}{|l|}{ Nombre et âge des enfants } \\
\hline Un seul enfant d'âge $\leq 6$ ans & $+123^{\star \star \star}$ & +87 & $+15^{\star}$ & +6 & $+139^{\star \star \star}$ & +87 \\
\hline Deux enfants et plus (au moins deux d'âge $\leq 6$ ans) & $+203^{\star \star \star}$ & +143 & $+42^{\star \star \star}$ & +18 & $+208^{\star \star \star}$ & +130 \\
\hline Deux enfants et plus (dont un d'âge $\leq 6$ ans) & $+100^{\star \star \star}$ & +71 & -1 & & $+127^{\star \star \star}$ & +80 \\
\hline Deux enfants et plus d'âge $>6$ ans & $+43^{\star \star \star}$ & +30 & -1 & & $+61^{\star \star \star}$ & +38 \\
\hline $\begin{array}{l}\text { Un seul enfant âgé de plus de } 6 \text { ans (et de moins } \\
\text { de } 18 \text { ans) }\end{array}$ & Réf. & & Réf. & & & \\
\hline \multicolumn{7}{|l|}{ Classes d'âge } \\
\hline$<35$ ans & $+25^{\star \star}$ & +17 & $+25^{\star \star \star}$ & +11 & $+19^{*}$ & +12 \\
\hline 35 à 44 ans & $+39^{\star \star \star}$ & +27 & $+32^{\star \star \star}$ & +14 & $+28^{\star \star \star}$ & +18 \\
\hline 45 ans et + & Réf. & & Réf. & & Réf. & \\
\hline \multicolumn{7}{|l|}{ Parent(s) enseignant(s) } \\
\hline Mère enseignante & +11 & & +1 & & $+16^{\star \star}$ & +10 \\
\hline Mère non enseignante & Réf. & & Réf. & & Réf. & \\
\hline Père enseignant & -12 & & +4 & & -17 & \\
\hline Père non enseignant & Réf. & & Réf. & & Réf. & \\
\hline \multicolumn{7}{|l|}{ Type de commune } \\
\hline Rurale à moins de 20000 hab. & Réf. & & Réf. & & Réf. & \\
\hline 20000 hab. à moins de 200000 hab. & +10 & & $+19^{\star \star \star}$ & +8 & +3 & \\
\hline 200000 hab. et + & $+19^{\star \star \star}$ & +13 & $+30^{\star \star \star}$ & +13 & -2 & \\
\hline
\end{tabular}

Note : ${ }^{\star \star \star}$ significatif à $1 \% ;{ }^{* \star}$ à $5 \%$; ${ }^{*}$ à $10 \% ;-:$ non significatif ; Réf. : catégorie de référence. Les effets marginaux sont précisés seulement quand le coefficient est significatif.

Lecture : un jour travaillé, les hommes enseignants consacrent 28 minutes de plus aux enfants que les hommes diplômés non enseignants.

Champ : enseignants et diplômés de l'enseignement supérieur (titulaires d'au moins une licence), ayant une activité professionnelle et vivant dans un ménage dans lequel il existe au moins un enfant de moins de 18 ans.

Source : enquête Emploi du temps 2010-2011. 
Tableau $\mathrm{E}$

Temps consacré aux enfants et au « noyau dur » par les femmes enseignantes en couple.

Résultats des estimations des modèles Tobit

(Référence : femme enseignante dont le conjoint est enseignant)

\begin{tabular}{|c|c|c|c|c|c|c|c|c|}
\hline $\begin{array}{l}\text { Coefficients, effets marginaux } \\
\text { et valeurs de } p\end{array}$ & \multicolumn{2}{|c|}{ Temps total } & \multicolumn{2}{|c|}{$\begin{array}{l}\text { Temps consacré aux } \\
\text { jeux et à l'instruction }\end{array}$} & \multicolumn{2}{|c|}{$\begin{array}{c}\text { Temps consacré aux } \\
\text { soins et à l'accompa- } \\
\text { gnement }\end{array}$} & \multicolumn{2}{|c|}{$\begin{array}{l}\text { Temps consacré au } \\
\text { noyau dur du travail } \\
\text { domestique }\end{array}$} \\
\hline Moyennes & \multirow{2}{*}{\multicolumn{2}{|c|}{$\begin{array}{l}103 \\
80 \%\end{array}$}} & \multirow{2}{*}{\multicolumn{2}{|c|}{$\begin{array}{c}25 \\
43 \%\end{array}$}} & \multirow{2}{*}{\multicolumn{2}{|c|}{$\begin{array}{c}78 \\
77 \%\end{array}$}} & \multirow{2}{*}{\multicolumn{2}{|c|}{$\begin{array}{l}123 \\
94 \%\end{array}$}} \\
\hline$\%$ concernées par l'activité & & & & & & & & \\
\hline & Coefficient & $\begin{array}{c}\text { Effet } \\
\text { marginal }\end{array}$ & Coefficient & $\begin{array}{c}\text { Effet } \\
\text { marginal }\end{array}$ & Coefficient & $\begin{array}{c}\text { Effet } \\
\text { marginal }\end{array}$ & Coefficient & $\begin{array}{c}\text { Effet } \\
\text { marginal }\end{array}$ \\
\hline \multicolumn{9}{|l|}{ Type de ménage } \\
\hline $\begin{array}{l}\text { En couple avec un } \\
\text { non-enseignant ayant } \\
\text { un diplôme <= Bac }\end{array}$ & $+28^{\star \star}$ & +21 & +9 & & $+28^{\star \star \star}$ & +20 & $-20^{\star \star}$ & -19 \\
\hline $\begin{array}{l}\text { En couple avec un } \\
\text { enseignant }\end{array}$ & Réf. & & Réf. & & Réf. & & Réf. & \\
\hline $\begin{array}{l}\text { En couple avec un } \\
\text { non-enseignant ayant un } \\
\text { diplôme de l'enseignement } \\
\text { supérieur }\end{array}$ & +17 & & $+22^{\star \star}$ & +9 & +6 & & $-15^{\star}$ & -14 \\
\hline \multicolumn{9}{|l|}{ Nombre et âge des enfants } \\
\hline $\begin{array}{l}\text { Un seul enfant d'âge } \\
\leq 6 \text { ans }\end{array}$ & $+131^{\star \star \star}$ & +99 & +21 & & $+122^{\star \star \star}$ & +86 & +13 & \\
\hline $\begin{array}{l}\text { Deux enfants et plus } \\
\text { (au moins deux d'âge } \\
\leq 6 \text { ans) }\end{array}$ & $+173^{\star \star \star}$ & +130 & $+74^{\star \star \star}$ & +31 & $+131^{\star \star \star}$ & +92 & +17 & \\
\hline $\begin{array}{l}\text { Deux enfants et plus (dont } \\
\text { un d'âge } \leq 6 \text { ans) }\end{array}$ & $+109^{\star * \star}$ & +82 & $+35^{\star \star \star}$ & +15 & $+98^{\star \star \star}$ & +69 & $+22^{*}$ & +20 \\
\hline $\begin{array}{l}\text { Deux enfants et plus d'âge } \\
>6 \text { ans }\end{array}$ & $+59^{\star \star \star}$ & +44 & $+23^{\star \star}$ & +10 & $+47^{\star \star \star}$ & +33 & $+21^{*}$ & +19 \\
\hline $\begin{array}{l}\text { Un seul enfant âgé de plus } \\
\text { de } 6 \text { ans (et de moins } \\
\text { de } 18 \text { ans) }\end{array}$ & Réf. & & Réf. & & Réf. & & Réf. & \\
\hline \multicolumn{9}{|l|}{ Classes d'âge } \\
\hline$<35$ ans & $+75^{\star \star \star}$ & +57 & $+53^{\star \star \star}$ & +22 & $+65^{\star \star \star}$ & +46 & -20 & \\
\hline 35 à 44 ans & $+68^{\star \star \star}$ & +51 & $+57^{\star \star \star}$ & +24 & $+53^{\star \star \star}$ & +37 & -1 & \\
\hline 45 ans et + & Réf. & & Réf. & & Réf. & & Réf. & \\
\hline \multicolumn{9}{|l|}{ Parent(s) enseignant(s) } \\
\hline Mère enseignante & -6 & & -14 & & 0 & & -11 & \\
\hline Mère non enseignante & Réf. & & Réf. & & Réf. & & Réf. & \\
\hline Père enseignant & +13 & & -15 & & +21 & & +15 & \\
\hline Père non enseignant & Réf. & & Réf. & & Réf. & & Réf. & \\
\hline \multicolumn{9}{|l|}{ Type de commune } \\
\hline $\begin{array}{l}\text { Rurale à moins } \\
\text { de } 20000 \text { hab. }\end{array}$ & Réf. & & Réf. & & Réf. & & Réf. & \\
\hline $\begin{array}{l}20000 \text { hab. à moins } \\
\text { de } 200000 \text { hab. }\end{array}$ & $+21^{*}$ & & $+28^{\star \star \star}$ & & +11 & & $-20^{\star \star}$ & -18 \\
\hline 200000 hab. et + & -4 & & +10 & & -8 & & -4 & \\
\hline \multicolumn{9}{|l|}{ Journée avec travail } \\
\hline Oui & -8 & & $-12^{*}$ & & +1 & & $-68^{\star \star \star}$ & -63 \\
\hline Non (ou moins de $2 h$ ) & Réf. & & Réf. & & Réf. & & Réf. & \\
\hline
\end{tabular}

Note : ${ }^{* *}$ significatif à $1 \% ;{ }^{* *}$ à $5 \%$; ${ }^{*}$ à $10 \% ;-:$ non significatif ; Réf. : catégorie de référence. Les effets marginaux sont précisés seulement quand le coefficient est significatif.

Lecture : les enseignantes dont le conjoint n'est pas enseignant et n'a pas de diplôme de l'enseignement supérieur consacrent 28 minutes de plus aux enfants que celles dont le conjoint est enseignant.

Champ : femmes enseignantes, en activité, vivant en couple dans un ménage dans lequel il existe au moins un enfant de moins de 18 ans.

Source : enquête Emploi du temps 2010-2011. 
Tableau F

Temps consacré aux enfants et au « noyau dur " par les hommes enseignants en couple.

Résultats des estimations des modèles Tobit

(Référence : homme enseignant dont la conjointe est enseignante)

\begin{tabular}{|c|c|c|c|c|c|c|c|c|}
\hline $\begin{array}{l}\text { Coefficients, effets marginaux } \\
\text { et valeurs de } p\end{array}$ & \multicolumn{2}{|c|}{ Temps total } & \multicolumn{2}{|c|}{$\begin{array}{l}\text { Temps consacré } \\
\text { aux jeux } \\
\text { et à l'instruction }\end{array}$} & \multicolumn{2}{|c|}{$\begin{array}{l}\text { Temps consacré } \\
\text { aux soins et à } \\
\text { l'accompagnement }\end{array}$} & \multicolumn{2}{|c|}{$\begin{array}{c}\text { Temps consacré } \\
\text { au noyau dur } \\
\text { du travail domestique }\end{array}$} \\
\hline Moyennes & \multirow{2}{*}{\multicolumn{2}{|c|}{$\begin{array}{c}55^{\prime} \\
67 \%\end{array}$}} & \multirow{2}{*}{\multicolumn{2}{|c|}{$\begin{array}{c}21 ' \\
31 \%\end{array}$}} & \multirow{2}{*}{\multicolumn{2}{|c|}{$\begin{array}{c}35 \\
54 \%\end{array}$}} & \multirow{2}{*}{\multicolumn{2}{|c|}{$\begin{array}{c}62 ' \\
82 \%\end{array}$}} \\
\hline$\%$ concernés par l'activité & & & & & & & & \\
\hline & Coefficient & $\begin{array}{c}\text { Effet } \\
\text { marginal }\end{array}$ & Coefficient & $\begin{array}{c}\text { Effet } \\
\text { marginal }\end{array}$ & Coefficient & $\begin{array}{c}\text { Effet } \\
\text { marginal }\end{array}$ & Coefficient & $\begin{array}{c}\text { Effet } \\
\text { marginal }\end{array}$ \\
\hline \multicolumn{9}{|l|}{ Type de ménage } \\
\hline $\begin{array}{l}\text { En couple avec une } \\
\text { non-enseignante ayant } \\
\text { un diplôme }<=\text { Bac }\end{array}$ & $+29^{\star \star}$ & +17 & $+51^{\star \star \star}$ & +15 & -3 & & -7 & \\
\hline $\begin{array}{l}\text { En couple avec une } \\
\text { enseignante }\end{array}$ & Réf. & & Réf. & & Réf. & & Réf. & \\
\hline $\begin{array}{l}\text { En couple avec une } \\
\text { non-enseignante ayant un } \\
\text { diplôme de l'enseignement } \\
\text { supérieur }\end{array}$ & +13 & & -1 & & +16 & & +5 & \\
\hline \multicolumn{9}{|l|}{ Nombre et âge des enfants } \\
\hline $\begin{array}{l}\text { Un seul enfant d'âge } \\
\leq 6 \text { ans }\end{array}$ & $+90^{\star \star \star}$ & +54 & $+63^{\star *}$ & +18 & $+75^{\star \star \star}$ & +37 & -1 & \\
\hline $\begin{array}{l}\text { Deux enfants et plus } \\
\text { (au moins deux d'âge } \\
\leq 6 \text { ans) }\end{array}$ & $+120^{\star \star \star}$ & +72 & $+60^{*}$ & +17 & $+113^{\star \star \star}$ & +56 & -11 & \\
\hline $\begin{array}{l}\text { Deux enfants et plus (dont } \\
\text { un d'âge } \leq 6 \text { ans) }\end{array}$ & $+96^{\star \star \star}$ & +58 & $+64^{\star \star \star}$ & +18 & $+81^{\star \star \star}$ & +40 & +8 & \\
\hline $\begin{array}{l}\text { Deux enfants et plus d'âge } \\
>6 \text { ans }\end{array}$ & $+50^{\star \star \star}$ & +30 & $+49^{\star \star \star}$ & +14 & $+28^{\star \star}$ & +14 & +5 & \\
\hline $\begin{array}{l}\text { Un seul enfant âgé de plus } \\
\text { de } 6 \text { ans (et de moins } \\
\text { de } 18 \text { ans) }\end{array}$ & Réf. & & Réf. & & Réf. & & Réf. & \\
\hline \multicolumn{9}{|l|}{ Classes d'âge } \\
\hline$<35$ ans & $+38^{*}$ & +23 & +9 & & +27 & & -1 & \\
\hline 35 à 44 ans & +21 & & +11 & & +17 & & +10 & \\
\hline 45 ans et + & Réf. & & Réf. & & Réf. & & Réf. & \\
\hline \multicolumn{9}{|l|}{ Parent(s) enseignant(s) } \\
\hline Mère enseignante & -16 & & -14 & & -3 & & -13 & \\
\hline Mère non enseignante & Réf. & & Réf. & & Réf. & & Réf. & \\
\hline Père enseignant & -15 & & $+35^{\star \star}$ & +10 & $-58^{\star \star \star}$ & -29 & -10 & \\
\hline Père non enseignant & Réf. & & Réf. & & Réf. & & Réf. & \\
\hline \multicolumn{9}{|l|}{ Type de commune } \\
\hline $\begin{array}{l}\text { Rurale à moins } \\
\text { de } 20000 \text { hab. }\end{array}$ & Réf. & & Réf. & & Réf. & & Réf. & \\
\hline $\begin{array}{l}20000 \text { hab. à moins } \\
\text { de } 200000 \text { hab. }\end{array}$ & $+25^{\star}$ & +15 & +3 & & $+35^{\star \star \star}$ & +17 & +7 & \\
\hline 200000 hab. et + & $+46^{\star \star \star}$ & $+27^{\star \star \star}$ & +12 & & $+39^{\star \star \star}$ & +19 & +12 & \\
\hline \multicolumn{9}{|l|}{ Journée avec travail } \\
\hline Oui & $+31^{\star \star \star}$ & +19 & $+42^{\star \star \star}$ & +12 & +11 & & $-33^{\star \star \star}$ & -26 \\
\hline Non (ou moins de $2 h$ ) & Réf. & & Réf. & & Réf. & & Réf. & \\
\hline
\end{tabular}

Note : ${ }^{* \star}$ significatif à $1 \% ;{ }^{* \star}$ à $5 \%$; ${ }^{*}$ à $10 \% ;-$ : non significatif ; Réf. : catégorie de référence. Les effets marginaux sont précisés seulement quand le coefficient est significatif.

Lecture : les enseignants dont la conjointe n'est pas enseignante et n'a pas de diplôme de l'enseignement supérieur consacrent 15 minutes de plus aux jeux et à l'instruction des enfants que ceux dont la conjointe est enseignante.

Champ : hommes enseignants, en activité, vivant en couple dans un ménage dans lequel il existe au moins un enfant de moins de 18 ans. Source : enquête Emploi du temps 2010-2011. 
Tableau G

Probabilité de faire partie de ceux ayant consacré le plus de temps aux enfants (Référence : femme diplômée non enseignante un jour travaillé)

\begin{tabular}{|c|c|c|}
\hline & Odds-ratio & Significativité \\
\hline \multicolumn{3}{|l|}{ Odds-ratio et valeurs de $p$} \\
\hline \multicolumn{3}{|l|}{ Sexe et profession } \\
\hline Femme enseignante un jour travaillé & 2,45 & *** \\
\hline Femme enseignante un jour non travaillé & 2,09 & ** \\
\hline Femme diplômée non enseignante un jour travaillé & Réf. & - \\
\hline Femme diplômée non enseignante un jour non travaillé & 2,78 & *** \\
\hline Homme enseignant un jour travaillé & 1,50 & n.s. \\
\hline Homme enseignant un jour non travaillé & 0,48 & n.s. \\
\hline Homme diplômé non enseignant un jour travaillé & 0,65 & ** \\
\hline Homme diplômé non enseignant un jour non travaillé & 1,44 & ** \\
\hline \multicolumn{3}{|l|}{ Type de ménage } \\
\hline Famille monoparentale & 1,61 & ** \\
\hline En couple avec enfant(s) et le conjoint n'est pas enseignant & Réf. & - \\
\hline En couple avec enfant(s) et le conjoint est enseignant & 1,08 & n.s. \\
\hline \multicolumn{3}{|l|}{ Nombre et âge des enfants } \\
\hline Un seul enfant d'âge $\leq 6$ ans & 5,87 & *** \\
\hline Deux enfants et plus (au moins deux d'âge $\leq 6$ ans) & 10,65 & *** \\
\hline Deux enfants et plus (dont un d'âge $\leq 6$ ans) & 4,14 & *** \\
\hline Deux enfants et plus d'âge $>6$ ans & 2,40 & *** \\
\hline Un seul enfant âgé de plus de 6 ans (et de moins de 18 ans) & Réf. & - \\
\hline \multicolumn{3}{|l|}{ Classes d'âge } \\
\hline$<35$ ans & 1,61 & ** \\
\hline 35 à 44 ans & 1,50 & ** \\
\hline 45 ans et + & Réf. & - \\
\hline \multicolumn{3}{|l|}{ Parent(s) enseignant(s) } \\
\hline Mère enseignante & 1,24 & n.s. \\
\hline Mère non enseignante & Réf. & - \\
\hline Père enseignant & 0,81 & n.s. \\
\hline Père non enseignant & Réf. & - \\
\hline \multicolumn{3}{|l|}{ Type de commune } \\
\hline Rurale à moins de 20000 hab. & Réf. & - \\
\hline 20000 hab. à moins de 200000 hab. & 1,10 & n.s. \\
\hline 200000 hab. et + & 1,21 & n.s. \\
\hline
\end{tabular}

Note : ${ }^{\star \star \star}$ significatif à $1 \% ;{ }^{\star \star}$ à $5 \%$; ${ }^{*}$ à $10 \%$; ns : non significatif ; Réf. : catégorie de référence.

Lecture : un odds-ratio supérieur à 1 (resp. inférieur à 1) et statistiquement significatif indique que l'on est en présence d'un facteur qui accroît (resp. diminue) les chances que l'évènement ait eu lieu. Plus l'odds-ratio est éloigné de 1, plus l'influence du facteur auquel il est associé est importante.

Champ : enseignants et diplômés de l'enseignement supérieur (titulaires d'au moins une licence), ayant une activité professionnelle et vivant dans un ménage dans lequel il existe au moins un enfant de moins de 18 ans.

Source : enquête Emploi du temps 2010-2011. 
Tableau $\mathrm{H}$

Probabilité de faire partie de ceux ayant consacré le plus de temps aux enfants (Référence : homme diplômé non enseignant un jour travaillé)

\begin{tabular}{|c|c|c|}
\hline & Odds-ratio & Significativité \\
\hline \multicolumn{3}{|l|}{ Odds-ratio et valeurs de $p$} \\
\hline \multicolumn{3}{|l|}{ Sexe et profession } \\
\hline Femme enseignante un jour travaillé & 3,78 & *** \\
\hline Femme enseignante un jour non travaillé & 3,22 & *** \\
\hline Femme diplômée non enseignante un jour travaillé & 1,54 & *** \\
\hline Femme diplômée non enseignante un jour non travaillé & 4,28 & *** \\
\hline Homme enseignant un jour travaillé & 2,31 & ** \\
\hline Homme enseignant un jour non travaillé & 0,74 & n.s. \\
\hline Homme diplômé non enseignant un jour travaillé & Réf. & - \\
\hline Homme diplômé non enseignant un jour non travaillé & 2,21 & *** \\
\hline \multicolumn{3}{|l|}{ Type de ménage } \\
\hline Famille monoparentale & 1,61 & ** \\
\hline En couple avec enfant(s) et le conjoint n'est pas enseignant & Réf. & - \\
\hline En couple avec enfant(s) et le conjoint est enseignant & 1,08 & n.s. \\
\hline \multicolumn{3}{|l|}{ Nombre et âge des enfants } \\
\hline Un seul enfant d'âge $\leq 6$ ans & 5,87 & *** \\
\hline Deux enfants et plus (au moins deux d'âge $\leq 6$ ans) & 10,65 & *** \\
\hline Deux enfants et plus (dont un d'âge $\leq 6$ ans) & 4,14 & *** \\
\hline Deux enfants et plus d'âge $>6$ ans & 2,40 & *** \\
\hline Un seul enfant âgé de plus de 6 ans (et de moins de 18 ans) & Réf. & - \\
\hline \multicolumn{3}{|l|}{ Classes d'âge } \\
\hline$<35$ ans & 1,61 & ** \\
\hline 35 à 44 ans & 1,50 & ** \\
\hline 45 ans et + & Réf. & - \\
\hline \multicolumn{3}{|l|}{ Parent(s) enseignant(s) } \\
\hline Mère enseignante & 1,24 & n.s. \\
\hline Mère non enseignante & Réf. & - \\
\hline Père enseignant & 0,81 & n.s. \\
\hline Père non enseignant & Réf. & - \\
\hline \multicolumn{3}{|l|}{ Type de commune } \\
\hline Rurale à moins de 20000 hab. & Réf. & - \\
\hline 20000 hab. à moins de 200000 hab. & 1,10 & n.s. \\
\hline 200000 hab. et + & 1,21 & n.s. \\
\hline
\end{tabular}

Note : ${ }^{\star \star \star}$ significatif à $1 \% ;{ }^{\star \star}$ à $5 \%$; ${ }^{*}$ à $10 \%$; ns : non significatif ; Réf. : catégorie de référence.

Lecture : un odds-ratio supérieur à 1 (resp. inférieur à 1) et statistiquement significatif indique que l'on est en présence d'un facteur qui accroît (resp. diminue) les chances que l'évènement ait eu lieu. Plus l'odds-ratio est éloigné de 1, plus l'influence du facteur auquel il est associé est importante.

Champ : enseignants et diplômés de l'enseignement supérieur (titulaires d'au moins une licence), ayant une activité professionnelle et vivant dans un ménage dans lequel il existe au moins un enfant de moins de 18 ans.

Source : enquête Emploi du temps 2010-2011. 
